\title{
The multi-dimensional super-replication problem under gamma constraints
}

\section{Problème de sur-réplication multi-dimensionnel sous contraintes gamma}

\author{
Patrick Cheridito $^{\mathrm{a}, 1}, \mathrm{H}$. Mete Soner ${ }^{\mathrm{b}, 2, *}$, Nizar Touzi $^{\mathrm{c}}$ \\ a ORFE, Princeton University, Princeton, NJ, USA \\ ${ }^{\mathrm{b}}$ Koç University, Istanbul, Turkey \\ ${ }^{\mathrm{c}}$ Centre de recherche en économie et statistique, Paris, France
}

Received 8 November 2003; accepted 27 October 2004

Available online 22 April 2005

\begin{abstract}
The classical Black-Scholes hedging strategy of a European contingent claim may require rapid changes in the replicating portfolio. One approach to avoid this is to impose a priori bounds on the variations of the allowed trading strategies, called gamma constraints. Under such a restriction, it is in general no longer possible to replicate a European contingent claim, and super-replication is a commonly used alternative. This paper characterizes the infimum of the initial capitals that allow an investor to super-replicate the contingent claim by carefully choosing an investment strategy obeying a gamma constraint. This infimum is shown to be the unique viscosity solution of a nonstandard partial differential equation. Due to the lower gamma bound, the "intuitive" partial differential equation is not parabolic and the actual equation satisfied by the infimum is the parabolic majorant of this equation. The derivation of the viscosity property is based on new results on the small time behavior of double stochastic integrals.
\end{abstract}

(C) 2005 L'Association Publications de l'Institut Henri Poincaré. Published by Elsevier B.V. All rights reserved

\section{Résumé}

La stratégie de couverture classique d'une option européenne, dictée par le modèle de Black et Scholes, peut conduire à des rebalancements rapides du portefeuille répliquant. Afin d'éviter de telles situations indésirables, nous introduisons des contraintes spécifiques sur le portefeuille, appelées contraintes gamma. Il n'est alors pas possible en général de répliquer

\footnotetext{
* Corresponding author.

E-mail addresses: dito@princeton.edu (P. Cheridito), cn0s@andrew.cmu.edu, msoner@ku.edu.tr (H.M. Soner), touzi@ensae.fr (N. Touzi).

1 Supported by the Swiss National Science Foundation and the Centre de Recherche en Economie et Statistique.

2 Member of the Turkish Academy of Sciences and this work was partly supported by the Turkish Academy of Sciences.
}

0294-1449/\$ - see front matter (C) 2005 L'Association Publications de l'Institut Henri Poincaré. Published by Elsevier B.V. All rights reserved doi:10.1016/j.anihpc.2004.10.012 
parfaitement l'option européene. Par conséquent, la surréplication est alors une alternative fréquemment utilisée. Dans ce papier, on caractérise l'infimum des capitaux initiaux qui permet à un investiseur de surrépliquer l'actif contingent en choisissant soigneusement une stratégie de portefeuile satisfaisant à une contrainte gamma. Nous montrons que cet infimum est l'unique solution de viscosité d'une équation aux dérivées partielles non standard. A cause de la borne inférieure sur la contrainte gamma, l'équation aux dérivées partielles «intuitive»n'est pas parabolique, et l'équation effectivemet satisfaite par l'infimum est le mojorant parabolique de l'équation «intuitive». L'obtention de la propriété de viscosité s'appuie sur des résultats nouveaux portant sur des intégrales stochastiques doubles.

(C) 2005 L'Association Publications de l'Institut Henri Poincaré. Published by Elsevier B.V. All rights reserved

MSC: 91B28; 35K55; 60H30

Keywords: Gamma constraints; Super-replication; Viscosity solutions; Parabolic majorant; Double stochastic integrals

Mots-clés : Contrainte gamma ; Sur-réplication ; Solutions de viscosité ; Majorant parabolique; Intégrales stochastiques doubles

\section{Introduction}

The classical Black-Scholes theory provides a mechanism for pricing and hedging contingent claims depending on a risky asset. In this framework it is assumed that the price of the risky asset follows a geometric Brownian motion

$$
\mathrm{d} S(t)=S(t)[\mu \mathrm{d} t+\sigma \mathrm{d} Z(t)]
$$

for a Brownian motion $Z$ and parameters $\mu \geqslant 0, \sigma>0$, and that apart from the risky asset, money can also be invested in a cash account, where it grows with a constant continuously compounded interest rate, which, without loss of generality, can be assumed to be zero. Then, investments in the cash account stay constant, and the price of the risky asset can be written as

$$
\mathrm{d} S(t)=S(t) \sigma \mathrm{d} W(t)
$$

where $W$ is a Brownian motion under a probability measure that is equivalent to the original one. For a finite time horizon $T>0, t \in[0, T]$ and $s \in(0, \infty)$, we denote by $\left\{S_{t, s}(r), t \leqslant r \leqslant T\right\}$ the solution to (1.1) with initial condition $S(t)=s$. Consider a European contingent claim with time $T$ payoff $g\left(S_{t, s}(T)\right)$ for a measurable function $g:(0, \infty) \rightarrow[0, \infty)$ that is dominated by a polynomial. The Black-Scholes price

$$
v^{\mathrm{BS}}(t, s)=\mathrm{E}\left[g\left(S_{t, s}(T)\right)\right]
$$

of the contingent claim is a smooth function of time $t \in[0, T)$ and the price of the underlying risky asset $s \in(0, \infty)$. The Black-Scholes hedging portfolio consists of $v_{s}^{\mathrm{BS}}(t, s)$ many shares of the risky asset and the amount of money $v^{\mathrm{BS}}(t, s)-v_{s}^{\mathrm{BS}}(t, s) s$ in the cash account. The value of this portfolio at maturity is equal to the payoff of the contingent claim, that is, the Black-Scholes hedging strategy replicates the contingent claim.

Several interesting constraints and deviations from the Black-Scholes model have been studied in the literature, for example, short-selling constraints or stochastic volatility; we refer to $[3,6,7,9-11,18]$ and the references therein. Under such constraints or imperfections, it is in general no longer possible to replicate a given contingent claim. Usually, one then tries to find a hedging portfolio that approximates the contingent claim in some sense or superreplicates it.

In this paper we study the super-replication problem of a European contingent claim under gamma constraints. We will allow the contingent claim to depend on several risky assets whose prices can have stochastic volatility. But to simplify the notation in this introduction, we here consider only one risky asset with price dynamics given 
by (1.1). In the Black-Scholes framework, the contingent claim's gamma is given by $v_{s s}^{\mathrm{BS}}(t, s)$. It gives the variation of $v_{s}^{\mathrm{BS}}(t, s)$ due to the variation of the underlying risky asset. Note that by Itô's lemma, for $r \in[t, T]$,

$$
v_{s}^{\mathrm{BS}}\left(r, S_{t, s}(r)\right)=v_{s}^{\mathrm{BS}}(t, s)+\int_{t}^{r} \mathcal{L} v_{s}^{\mathrm{BS}}\left(u, S_{t, s}(u)\right) \mathrm{d} u+\int_{t}^{r} v_{s s}^{\mathrm{BS}}\left(u, S_{t, s}(u)\right) \mathrm{d} S_{t, s}(u),
$$

where $\mathcal{L}$ is the Dynkin operator of $S_{t, s}$ given by

$$
\mathcal{L} \varphi(t, s)=\frac{\partial}{\partial t} \varphi(t, s)+\frac{1}{2} \sigma^{2} s^{2} \frac{\partial^{2}}{\partial s^{2}} \varphi(t, s) .
$$

Motivated by (1.2), we consider self-financing trading strategies such that the process describing the number of shares of the risky asset held at time $r \in[t, T]$ can be written as

$$
Y(r)=a(r)+\int_{t}^{r} \gamma(u) \mathrm{d} S_{t, s}(u)
$$

for a progressively measurable finite variation process $a$ and a progressively measurable process $\gamma$ such that the pair $(a, \gamma)$ satisfies certain boundedness conditions to be specified in Subsection 2.2. We then denote the associated trading strategy of the form (1.3) by $Y_{s, t}^{(a, \gamma)}$ and the corresponding wealth process with initial capital $x \in \mathbb{R}$ by

$$
X_{t, s, x}^{(a, \gamma)}(r)=x+\int_{t}^{r} Y_{t, s}^{(a, \gamma)}(u) \mathrm{d} S_{t, s}(u), \quad r \in[t, T] .
$$

Now, a gamma constraint can be expressed as a restriction on the process $\gamma$. In this paper we consider gamma constraints of the form

$$
\Gamma_{*} \leqslant S_{t, s}^{2}(r) \gamma(r) \leqslant \Gamma^{*}, \quad \text { for all } r \in[t, T],
$$

where $-\infty \leqslant \Gamma_{*}<\Gamma^{*} \leqslant \infty$ are two fixed constants. By $\mathcal{A}_{t, s}$ we denote the set of all pairs $(a, \gamma)$ that satisfy the above conditions for the initial condition $S(t)=s$ and some other technical conditions described in Subsection 2.2. In view of the approximation results of Leventhal and Skorokhod [13] and Bank and Baum [1], these technical conditions are very important. A detailed discussion is given in Remark 3.11 below.

The infimum of all initial capitals that allow to super-replicate the contingent claim is given by

$$
v(t, s):=\inf \left\{x: X_{t, s, x}^{(a, \gamma)}(T) \geqslant g\left(S_{t, s}(T)\right) \text { for some }(a, \gamma) \in \mathcal{A}_{t, s}\right\},
$$

where the inequality is understood in the almost sure sense. The purpose of this paper is to characterize the function $v$ as the unique viscosity solution of a partial differential equation together with a terminal condition. The equation will be derived from a dynamic programming principle (DPP). Therefore, we will refer to it as the dynamic programming equation (DPE) for $v$.

Note that if $g$ is twice continuously differentiable and satisfies the gamma constraint

$$
\Gamma_{*} \leqslant s^{2} g_{s s}(s) \leqslant \Gamma^{*} \text { for all } s \in(0, \infty),
$$

then also,

$$
\begin{aligned}
s^{2} v_{s s}^{\mathrm{BS}}(t, s) & =s^{2} \frac{\partial^{2}}{\partial s^{2}} \mathrm{E}\left[g\left(s \exp \left\{\sigma[W(T)-W(t)]-\frac{1}{2} \sigma^{2}[T-t]\right\}\right)\right] \\
& =\mathrm{E}\left[\left(S_{t, s}(T)\right)^{2} g_{s s}\left(S_{t, s}(T)\right)\right] \in\left[\Gamma_{*}, \Gamma^{*}\right]
\end{aligned}
$$


for all $s \in(0, \infty)$. Hence, it can be seen from (1.2) that the Black-Scholes strategy satisfies the gamma constraint and therefore, $v^{\mathrm{BS}}=v$. For functions $g$ that do not necessarily satisfy the gamma constraint (1.6), the DPE for $v$ derived in [15] for the one-dimensional case with $\Gamma_{*}=-\infty$ is

$$
\min \left\{-v_{t}-\frac{1}{2} \sigma^{2} s^{2} v_{s s} ; \Gamma^{*}-s^{2} v_{s s}\right\}=0 .
$$

(In fact, in [15], the gamma constraint $s v_{s s} \leqslant \Gamma^{*}$ is considered, which leads to a DPE slightly different from (1.7). However, if the arguments of [15] are adapted to the gamma constraint (1.4), one gets (1.7).) Eq. (1.7) agrees with the intuition that the solution of the problem consists in forgetting about the constraint as long as it is satisfied, and a free boundary behavior whenever the constraint binds.

The same kind of reasoning, leads us to guess that the DPE associated to (1.5) (with $\Gamma_{*}>-\infty$ ) is

$$
F\left(s, v_{t}, v_{s s}\right):=\min \left\{-v_{t}-\frac{1}{2} \sigma^{2} s^{2} v_{s s} ; \Gamma^{*}-s^{2} v_{s s} ; s^{2} v_{s s}-\Gamma_{*}\right\}=0 .
$$

However, one immediately observes that $F\left(s, v_{t}, v_{s s}\right)$ is not monotone in $v_{s s}$. Hence, we do not expect the above equation to be the correct one, and indeed, it is not.

It follows from Theorem 3.6 below that the function $v$ is a viscosity solution of the equation

$$
\widehat{F}\left(s, v_{t}(t, s), v_{s s}(t, s)\right)=0,
$$

where $\widehat{F}(s, p, A)$ is the smallest function $\phi \geqslant F$ which is decreasing in the $A$ variable. We give the following example to illustrate this point:

Example 1.1. Consider the problem in one dimension with $\Gamma^{*}=+\infty, \Gamma_{*}=0, \sigma \equiv 1$, and with $g(s)=s \wedge 1$ for $s \in(0, \infty)$. Then,

$$
F\left(s, v_{t}, v_{s s}\right)=\min \left\{-v_{t}-\frac{1}{2} s^{2} v_{s s} ; s^{2} v_{s s}\right\} .
$$

Notice that any function $v$ satisfying $F\left(s, v_{t}, v_{s s}\right)=0$ in the viscosity sense also satisfies $v_{s s} \geqslant 0$ in the viscosity sense and is therefore convex. However, we claim that the minimal super-replicating cost $v$ in this example is equal to $g$. In particular, it is not convex. Indeed, consider the following strategy: if $s \leqslant 1$, then use initial capital $x=s$ and buy and hold one share of the risky asset. The resulting terminal wealth is $X_{T}=S_{T} \geqslant g\left(S_{T}\right)$. If $s \geqslant 1$, then with $x=1$ buy no shares of the risky asset and hold the money in the cash account. This leads to a terminal wealth of $X_{T}=1 \geqslant g\left(S_{T}\right)$. Hence, this strategy with $x=g(s)$ is super-replicating and therefore, $v(s, t) \leqslant g(s)$ for all $(t, s) \in[0, T) \times(0, \infty)$.

The opposite inequality and consequently $v=g$ follows from Theorem 3.6 and the fact that $g$ is a viscosity solution of the equation $\widehat{F}\left(s, v_{t}, v_{s s}\right)=0$. Notice that $v=g$ is not a viscosity supersolution of $s^{2} v_{s s}=0$. So, we see in this example that $v$ need not be a viscosity solution of Eq. (1.9). Also, $v$ is in general not differentiable, and $v_{s s}$ does not necessarily satisfy the gamma constraint (1.4) in the viscosity sense.

In the literature, super-replication problems under constraints are usually approached by duality. Then, the dual formulation turns out to be in the standard form, and it is straightforward to arrive at the associated DPE. However, the usual techniques from this literature do not apply in our setup, and we are currently not able to derive the correct DPE for the problem (1.5) via convex duality techniques. In this paper, we continue with the method developed by Soner and Touzi in [15], and later in [16] and [17], in order to derive the DPE for (1.5). The main ingredients in this derivation are the two partial dynamic programming principles presented in Subsections 4.2 and 5.2 and a precise analysis of the small time behavior of double stochastic integrals. This analysis is carried out in the accompanying paper [4]. The results from [4] that are needed in this paper are reported in the Appendix. 
Notation. Equalities and inequalities between random variables are understood to hold in the almost sure sense. By $\mathcal{M}^{d}$ we denote the set of all $d \times d$ matrices with real coefficients. $A^{T}$ is the transpose of a matrix $A \in \mathcal{M}^{d}$ and $\operatorname{Tr}[A]$ its trace. The set $\mathcal{S}^{d}$ is the collection of all symmetric matrices of $\mathcal{M}^{d}$. The subset of positive semi-definite symmetric matrices will be denoted by $\mathcal{S}_{+}^{d}$. For $x \in \mathbb{R}^{d}$, we set

$$
|x|:=\sqrt{x_{1}^{2}+\cdots+x_{d}^{2}}
$$

and for $A \in \mathcal{M}^{d}$,

$$
|A|:=\sup _{x \in \mathbb{R}^{d},|x| \leqslant 1} A x .
$$

For a vector $x \in \mathbb{R}^{d}, \operatorname{diag}[x]$ is the $d \times d$-diagonal matrix with diagonal elements $x_{1}, \ldots, x_{d}$.

For a function $v$ on a subset $Q$ of $\mathbb{R}^{n}$, we denote by $v^{*}$ and $v_{*}$ the functions on $\bar{Q}$ given by

$$
v^{*}(x)=\lim _{r \searrow 0} \sup _{y \in Q,|y-x| \leqslant r} v(y), \quad x \in \bar{Q},
$$

and

$$
v_{*}(x)=\lim _{r \searrow 0} \inf _{y \in Q,|y-x| \leqslant r} v(y), \quad x \in \bar{Q},
$$

respectively. $v^{*}$ is the smallest upper semicontinuous function majorizing $v$ and is called the upper semicontinuous envelope of $v . v_{*}$ is the largest lower semicontinuous function minorizing $v$ and is called the lower semicontinuous envelope of $v$.

\section{Problem formulation}

In this section, we describe the model and the admissible trading strategies.

\subsection{Model}

We consider a financial market which consists of a cash account and $d$ risky assets. Since we are interested in almost sure super-replication, it is enough to specify the price dynamics under a risk neutral probability measure $P$. Let $T>0$ be a finite time horizon and $\{W(t), 0 \leqslant t \leqslant T\}$ a $d$-dimensional Brownian motion on a complete probability space $(\Omega, \mathcal{F}, P)$. By $\mathbb{F}^{W}$ we denote the smallest filtration $\left\{\mathcal{F}^{W}(t), 0 \leqslant t \leqslant T\right\}$ that contains the filtration generated by $\{W(t), 0 \leqslant t \leqslant T\}$ and satisfies the usual conditions. We take the cash account as numéraire and assume that the prices of the risky assets evolve according to the stochastic differential equation

$$
\mathrm{d} S(r)=\operatorname{diag}[S(r)] \sigma(S(r)) \mathrm{d} W(r),
$$

where $\sigma$ is a Lipschitz-continuous, bounded, $\mathcal{M}^{d}$-valued mapping defined on $(0, \infty)^{d}$ such that $\sigma(s)$ is invertible for all $s \in(0, \infty)^{d}$. By Exercise IX.2.10 of [14], the SDE (2.1) has for all initial conditions

$$
S(t)=s, \quad(t, s) \in[0, T] \times(0, \infty)^{d},
$$

a unique strong solution, which we denote by $S_{t, s}$. Note that every component $S_{t, s, i}$ of $S_{t, s}$ satisfies

$$
S_{t, s, i}(r)=s_{i} \exp \left(\sum_{j=1}^{d}\left[\int_{t}^{r} \sigma_{i j}\left(S_{t, s}(u)\right) \mathrm{d} W^{j}(u)-\frac{1}{2} \int_{t}^{r} \sigma_{i j}^{2}\left(S_{t, s}(u)\right) \mathrm{d} u\right]\right) .
$$


From this and Doob's $L^{p}$-inequality (see e.g. Theorem II.1.7 in [14]), it can be deduced that $S_{t, s}$ is a martingale that satisfies

$$
\mathrm{E}\left[\sup _{t \leqslant r \leqslant T}\left|S_{t, s}(r)\right|^{p}\right]<\infty, \quad \text { for all } p \geqslant 0 .
$$

Throughout this paper, we fix a parameter $\beta \geqslant 0$, and for an $\mathbb{F}^{W}$-progressively measurable process $\{H(r), t \leqslant$ $r \leqslant T\}$ taking values in $\mathbb{R}, \mathbb{R}^{d}$ or $\mathcal{M}^{d}$, we define

$$
\|H\|_{t, s}^{\beta, \infty}:=\left\|\sup _{t \leqslant r \leqslant T} \frac{|H(r)|}{1+\left|S_{t, s}(r)\right|^{\beta}}\right\|_{\mathbb{L}^{\infty}} .
$$

\subsection{Trading strategies and gamma constraints}

Consider an economic agent that starts at time $t$ with initial capital $x$ and holds $Y^{i}(r)$ shares of the $i$-th risky asset at time $r \in[t, T]$. Assume that the stochastic integral $\int_{t}^{r} Y(u)^{T} \mathrm{~d} S_{t, s}(u), r \in[t, T]$, exists and trading is done in a self-financing way. Then, the evolution of the economic agent's wealth is given by

$$
X(r)=x+\int_{t}^{r} Y(u)^{T} \mathrm{~d} S_{t, s}(u), \quad r \in[t, T] .
$$

To introduce constraints on the variations of investment strategies $Y$, we require them to be of the form

$$
Y(r)=\sum_{n=0}^{N-1} y^{n} \mathbf{1}_{\left\{\tau_{n} \leqslant r<\tau_{n+1}\right\}}+\int_{t}^{r} \alpha(u) \mathrm{d} u+\int_{t}^{r} \gamma(u) \mathrm{d} S_{t, s}(u),
$$

where $\|Y\|_{t, S}^{\beta, \infty}<\infty ; t=\tau_{0} \leqslant \tau_{1} \leqslant \cdots$ is an increasing sequence of $[t, T]$-valued $\mathbb{F}^{W}$-stopping times such that the random variable $N:=\inf \left\{n \in \mathbb{N}: \tau_{n}=T\right\}$ is bounded; $y^{n}$ is an $\mathbb{R}^{d}$-valued, $\mathcal{F}^{W}\left(\tau_{n}\right)$-measurable random variable satisfying $y^{n} \mathbf{1}_{\left\{\tau_{n}=T\right\}}=0 ; \alpha$ is an $\mathbb{R}^{W}$-progressively measurable, $\mathbb{R}^{d}$-valued process such that $\|\alpha\|_{t, s}^{\beta, \infty}<\infty$; and $\gamma$ is an $\mathcal{S}^{d}$-valued stochastic process such that $\|\gamma\|_{t, s}^{\beta, \infty}<\infty$ and the $i j$ component of $\gamma$ is of the form

$$
\gamma_{i j}(r)=\sum_{n=0}^{N-1} z_{i j}^{n} \mathbf{1}_{\left\{\tau_{n} \leqslant r<\tau_{n+1}\right\}}+\int_{t}^{r} \gamma_{i j}^{1}(u) \mathrm{d} u+\int_{t}^{r} \gamma_{i j}^{2}(u)^{T} \mathrm{~d} W(u),
$$

where $z_{i j}^{n}$ is an $\mathcal{F}^{W}\left(\tau_{n}\right)$-measurable random variable satisfying $z_{i j}^{n} \mathbf{1}_{\left\{\tau_{n}=T\right\}}=0 ; \gamma_{i j}^{1}$ is an $\mathbb{R}^{W}$-progressively measurable, $\mathbb{R}$-valued process such that $\left\|\gamma_{i, j}^{1}\right\|_{t, s}^{\beta, \infty}<\infty$; and $\gamma_{i j}^{2}$ is an $\mathbb{F}^{W}$-progressively measurable process taking values in $\mathbb{R}^{d}$ such that $\left\|\gamma_{i, j}^{2}\right\|_{t, s}^{\beta, \infty}<\infty$.

Under these assumptions, a trading strategy is determined by the choice of the control $v:=\left(\left(\tau_{n}, y^{n}\right)_{n} \geqslant 0, \alpha, \gamma\right)$. The set of admissible controls $\mathcal{A}_{t, s}$ is the collection of all such controls which in addition obey to the following gamma constraint:

$$
\operatorname{diag}\left[S_{t, s}(r)\right] \gamma(r) \operatorname{diag}\left[S_{t, s}(r)\right] \in K \quad \text { for all } t \leqslant r \leqslant T,
$$

where $K$ is a closed, convex, strict subset of $\mathcal{S}^{d}$ such that

$$
0 \in \operatorname{int}(K) \text {. }
$$

The boundedness conditions imposed on the strategies $Y$ are crucial for the proof of the viscosity supersolution property in Section 5. Without these conditions our main results, Theorems 3.6 and 3.7 do not hold true. More details are given in Remark 3.11. 
By

$$
\left\{\left(X_{t, s, x}^{v}, Y_{t, s}^{v}\right)(r), t \leqslant r \leqslant T\right\}
$$

we denote the process defined by the dynamics (2.3) and (2.4), the initial condition $X_{t, s, x}^{v}(t)=x$, and the control $v \in \mathcal{A}_{t, s}$. It can easily be deduced from (2.2) and the special form of our controls that $X_{t, s, x}^{\nu}$ is a square integrable martingale for all $(t, s, x) \in[0, T] \times(0, \infty)^{d} \times \mathbb{R}$ and $v \in \mathcal{A}_{t, s}$. In particular,

$$
x=\mathrm{E}\left[X_{t, s, x}^{v}(T)\right] .
$$

\subsection{Value function}

We now are in a position to define the stochastic control problem of interest. Consider a European contingent claim with time $T$ payoff $g\left(S_{t, s}(T)\right)$ for some lower semicontinuous function $g:(0, \infty) \rightarrow[0, \infty)$. The value function $v$ of the super-replication problem of $g$ under the gamma constraint (2.6) is given by

$$
v(t, s):=\inf \left\{x \in \mathbb{R}: X_{t, s, x}^{v}(T) \geqslant g\left(S_{t, s}(T)\right) \text { for some } v \in \mathcal{A}_{t, s}\right\},
$$

where we set $\inf \emptyset:=\infty$.

Remark 2.1. For $(t, s) \in[0, T) \times(0, \infty)^{d}$, let $x \in \mathbb{R}$ and $v \in \mathcal{A}_{t, s}$ such that

$$
X_{t, s, x}^{v}(T) \geqslant g\left(S_{t, s}(T)\right) .
$$

By (2.8),

$$
x=\mathrm{E}\left[X_{t, s, x}^{v}(T)\right] \geqslant \mathrm{E}\left[g\left(S_{t, s}(T)\right)\right] .
$$

This shows that

$$
v(t, s) \geqslant \mathrm{E}\left[g\left(S_{t, s}(T)\right)\right] \geqslant 0 \quad \text { for all }(t, s) \in[0, T) \times(0, \infty)^{d} .
$$

\section{Main results}

\subsection{Operators}

We start by introducing the operators that will be used in our analysis. The Dynkin operator associated to the process $S$ is given by

$$
\mathcal{L} \varphi(t, s):=-L\left(s, \varphi_{t}(t, s), D^{2} \varphi(t, s)\right)
$$

where $D^{2}$ denotes the second derivative with respect to the $s$-variables and $L$ is the parabolic operator

$$
L(s, p, A):=-p-\frac{1}{2} \operatorname{Tr}\left[\sigma(s)^{T} \operatorname{diag}[s] A \operatorname{diag}[s] \sigma(s)\right] .
$$

To express the constraint (2.6) as an inequality, we define for $A \in \mathcal{S}^{d}$ the signed distance of $A$ to the complement of $K$ in $\mathcal{S}^{d}$ :

$$
H(A):= \begin{cases}\inf \left\{|A-B|: B \in \mathcal{S}^{d} \backslash K\right\}, & \text { if } A \in K, \\ -\inf \{|A-B|: B \in K\}, & \text { if } A \in \mathcal{S}^{d} \backslash K .\end{cases}
$$

Since $K$ is a non-empty, strict subset of $\mathcal{S}^{d},-\infty<H(A)<\infty$ for all $A \in \mathcal{S}^{d}$. It is clear that $A$ is in $K$ if and only if $H(A) \geqslant 0$, and $A$ is in the interior of $K$ if and only if $H(A)>0$. Furthermore, it follows from the convexity of $K$ that $H$ is concave. 
With this notation the $d$-dimensional analog of the operator in (1.8) can be written as

$$
F(s, p, A):=\min \{L(s, p, A), H(\operatorname{diag}[s] A \operatorname{diag}[s])\} .
$$

Note that $F(s, p, A)$ is concave in $(p, A)$ because the mappings $(p, A) \mapsto L(s, p, A)$ and $(p, A) \mapsto H(A)$ are so. On the other hand, $F(s, p, A)$ is in general not monotone in $A$. Simply consider the following example:

Example 3.1. Let $0 \leqslant \Gamma^{*} \leqslant \infty$ and $-\infty \leqslant \Gamma_{*} \leqslant 0$ be two symmetric matrices such that all eigenvalues of $\Gamma^{*}-\Gamma_{*}$ are strictly positive. Then,

$$
K=\left[\Gamma_{*}, \Gamma^{*}\right]:=\left\{A \in \mathcal{S}^{d}: \Gamma_{*} \leqslant A \leqslant \Gamma^{*}\right\}
$$

is a closed convex subset of $\mathcal{S}^{d}$ that contains 0 in its interior. $\Gamma_{*}=-\infty$ (resp. $\left.\Gamma^{*}=\infty\right)$ means that there is no lower (resp. upper) constraint on the control process $\gamma$. If $d=1$ and $-\infty<\Gamma_{*}<\Gamma^{*}<\infty$, then

$$
H(A)=\min \left\{\Gamma^{*}-A, A-\Gamma_{*}\right\},
$$

and $F(s, p, A)$ is not monotone in $A$.

\subsection{Parabolic envelope}

For any function $\phi: \mathcal{S}^{d} \rightarrow \mathbb{R}$, we define the function $\hat{\phi}: \mathcal{S}^{d} \rightarrow(-\infty, \infty]$ as follows:

$$
\hat{\phi}(A):=\sup _{B \in \mathcal{S}_{+}^{d}} \phi(A+B) .
$$

Lemma 3.2. $\hat{\phi}$ is the smallest decreasing majorant of $\phi$.

Proof. 1. The inequality $\hat{\phi} \geqslant \phi$ follows from the fact that $0 \in \mathcal{S}_{+}^{d}$.

2. Now, let $A \geqslant A^{\prime}$ be two ordered matrices in $\mathcal{S}^{d}$. Then, $\mathcal{S}_{+}^{d} \subset \mathcal{S}_{+}^{d}+\left(A^{\prime}-A\right)$, and therefore,

$$
\hat{\phi}\left(A^{\prime}\right)=\sup _{B \in \mathcal{S}_{+}^{d}} \phi\left(A+\left(A^{\prime}-A\right)+B\right)=\sup _{B \in \mathcal{S}_{+}^{d}+\left(A^{\prime}-A\right)} \phi(A+B) \geqslant \sup _{B \in \mathcal{S}_{+}^{d}} \phi(A+B)=\hat{\phi}(A) .
$$

3. Let $\tilde{\phi}$ be a decreasing mapping from $\mathcal{S}^{d}$ to $(-\infty, \infty]$ such that $\tilde{\phi} \geqslant \phi$. Then, for all $A \in \mathcal{S}^{d}$ and $B \in \mathcal{S}_{+}^{d}$, we have $\tilde{\phi}(A) \geqslant \tilde{\phi}(A+B) \geqslant \phi(A+B)$. Hence, $\tilde{\phi}(A) \geqslant \sup _{B \in \mathcal{S}_{+}^{d}} \phi(A+B)=\hat{\phi}(A)$.

For a function $\phi:(0, \infty)^{d} \times \mathbb{R} \times \mathcal{S}^{d} \rightarrow \mathbb{R}$, we define $\hat{\phi}$ by

$$
\hat{\phi}(s, p, A):=\sup _{B \in \mathcal{S}_{+}^{d}} \phi(s, p, A+B) .
$$

Lemma 3.3. Let $s \in(0, \infty)^{d}$. If $\phi(s, \cdot, \cdot)$ is concave, then so is $\hat{\phi}(s, \cdot, \cdot)$.

Proof. First assume that there exists a pair $\left(p_{1}, A_{1}\right) \in \mathbb{R} \times \mathcal{S}^{d}$ such that $\hat{\phi}\left(s, p_{1}, A_{1}\right)=\infty$. For every $(p, A) \in$ $\mathbb{R} \times \mathcal{S}^{d}$, there exists a $\left(p_{2}, A_{2}\right) \in \mathbb{R} \times \mathcal{S}^{d}$ such that

$$
(p, A)=\frac{1}{2}\left(p_{1}, A_{1}\right)+\frac{1}{2}\left(p_{2}, A_{2}\right)
$$

By the definition of $\hat{\phi}$ and the concavity of $\phi(s, \cdot, \cdot)$,

$$
\hat{\phi}(s, p, A) \geqslant \phi\left(s, p, A+\frac{1}{2} B\right) \geqslant \frac{1}{2} \phi\left(s, p_{1}, A_{1}+B\right)+\frac{1}{2} \phi\left(s, p_{2}, A_{2}\right)
$$


for all $B \in \mathcal{S}_{+}^{d}$, which implies that $\hat{\phi}(s, p, A)=\infty$. Hence, $\hat{\phi}(s, p, A)=\infty$ for all $(p, A) \in \mathbb{R} \times \mathcal{S}^{d}$. In particular, $\hat{\phi}(s, \cdot, \cdot)$ is concave.

If $\hat{\phi}(s, p, A)<\infty$ for all $(p, A) \in \mathbb{R} \times \mathcal{S}^{d}$, consider two pairs $\left(p_{1}, A_{1}\right)$ and $\left(p_{2}, A_{2}\right)$ in $\mathbb{R} \times \mathcal{S}^{d}$, and let $\lambda \in(0,1)$. For all $\varepsilon>0$, there exist $B_{1}^{\varepsilon}$ and $B_{2}^{\varepsilon}$ in $\mathcal{S}_{+}^{d}$ such that

$$
\hat{\phi}\left(p_{i}, A_{i}\right) \leqslant \phi\left(p_{i}, A_{i}+B_{i}^{\varepsilon}\right)+\varepsilon \text { for } i=1,2 .
$$

Then,

$$
\begin{aligned}
\lambda \hat{\phi}\left(s, p_{1}, A_{1}\right)+(1-\lambda) \hat{\phi}\left(s, p_{2}, A_{2}\right) & \leqslant \lambda \phi\left(s, p_{1}, A_{1}+B_{1}^{\varepsilon}\right)+(1-\lambda) \phi\left(s, p_{2}, A_{2}+B_{2}^{\varepsilon}\right)+\varepsilon \\
& \leqslant \phi\left(s, \lambda p_{1}+(1-\lambda) p_{2}, \lambda\left(A_{1}+B_{1}^{\varepsilon}\right)+(1-\lambda)\left(A_{2}+B_{2}^{\varepsilon}\right)\right)+\varepsilon \\
& \leqslant \hat{\phi}\left(s, \lambda p_{1}+(1-\lambda) p_{2}, \lambda A_{1}+(1-\lambda) A_{2}\right)+\varepsilon,
\end{aligned}
$$

and the required result is obtained by sending $\varepsilon$ to zero.

Since $F(s, p, A) \leqslant L(s, p, A)$ and $L(s, p, A)$ is decreasing in $A$, it follows from Lemma 3.2 that $\widehat{F}(s, p, A) \leqslant$ $L(s, p, A)$ and $\widehat{F}$ is the smallest function above $F$ that is monotone in $A$. Therefore, we call it the parabolic envelope of $F$. By Lemma 3.3, $\widehat{F}(s, \cdot, \cdot)$ inherits the concavity from $F(s, \cdot, \cdot)$.

Lemma 3.4. The mapping

$$
\widehat{F}:(0, \infty)^{d} \times \mathbb{R} \times \mathcal{S}^{d} \rightarrow \mathbb{R}
$$

is continuous.

Proof. 1. Lower semicontinuity: Let $\left(s_{0}, p_{0}, A_{0}\right) \in(0, \infty)^{d} \times \mathbb{R} \times \mathcal{S}^{d}$ and $\varepsilon>0$. By definition of $\widehat{F}$ and continuity of $F$, there exists a $B_{0} \in \mathcal{S}_{+}^{d}$ and a neighborhood $U$ of $\left(s_{0}, p_{0}, A_{0}\right)$ such that for all $(s, p, A) \in U$,

$$
\widehat{F}\left(s_{0}, p_{0}, A_{0}\right)-\varepsilon \leqslant F\left(s_{0}, p_{0}, A_{0}+B_{0}\right)-\frac{\varepsilon}{2} \leqslant F\left(s, p, A+B_{0}\right) \leqslant \widehat{F}(s, p, A),
$$

which proves that $\widehat{F}$ is lower semicontinuous.

2. Upper semicontinuity: Let $\left(s_{k}, p_{k}, A_{k}\right)_{k} \geqslant 1$ be a sequence in $(0, \infty)^{d} \times \mathbb{R} \times \mathcal{S}^{d}$ converging to a point $\left(s_{0}, p_{0}, A_{0}\right) \in(0, \infty)^{d} \times \mathbb{R} \times \mathcal{S}^{d}$. There exists for all $k \geqslant 1$, a $B_{k} \in \mathcal{S}_{+}^{d}$ such that

$$
F\left(s_{k}, p_{k}, A_{k}+B_{k}\right) \geqslant \widehat{F}\left(s_{k}, p_{k}, A_{k}\right)-\frac{1}{k} \geqslant F\left(s_{k}, p_{k}, A_{k}\right)-\frac{1}{k} .
$$

Since $F\left(s_{k}, p_{k}, A_{k}\right)-1 / k$ converges to $F\left(s_{0}, p_{0}, A_{0}\right)$ and $\sigma\left(s_{0}\right)$ is assumed to be invertible, it follows from the definition of $F$ (3.3) and the form of $L$ (3.1) that the sequence $\left(B_{k}\right)_{k} \geqslant 1$ is bounded. Hence, there exists a subsequence $B_{k_{j}}$ that converges to a $B_{0} \in \mathcal{S}_{+}^{d}$. Then,

$$
\limsup _{j \rightarrow \infty} \widehat{F}\left(s_{k_{j}}, p_{k_{j}}, A_{k_{j}}\right) \leqslant \limsup _{k \rightarrow \infty} F\left(s_{k_{j}}, p_{k_{j}}, A_{k_{j}}+B_{k_{j}}\right)=F\left(s_{0}, p_{0}, A_{0}+B_{0}\right) \leqslant \widehat{F}\left(s_{0}, p_{0}, A_{0}\right) .
$$

This shows that $\widehat{F}$ is upper semicontinuous.

\subsection{Equation}

Earlier results indicate that the value function $v$ is a viscosity solution of the equation

$$
\widehat{F}\left(s, v_{t}(t, s), D^{2} v(t, s)\right)=0 \quad \text { for }(t, s) \in[0, T) \times(0, \infty)^{d},
$$

where $\widehat{F}$ is the parabolic majorant of the function $F$ defined in (3.3). 
This equation has to be complemented with an appropriate terminal condition. In the next subsections, we will describe the terminal behavior and state the main results.

Note that the process $S$ never reaches the lateral boundary. For this reason, we do not need to specify lateral boundary conditions.

\subsection{Terminal condition}

It has already been observed in the literature that constraints on the hedging strategy can lead to the situation that the limit

$$
\lim _{t^{\prime} \nearrow T, s^{\prime} \rightarrow s} v\left(t^{\prime}, s^{\prime}\right)
$$

exists but does not coincide with the function $g$. We refer to [3] and [7] for the case of portfolio constraints and to [15] for the case of an upper gamma bound. The reason for this phenomenon is the following: The gamma constraint induces restrictions on the function $v(t, s)$ on $[0, T) \times(0, \infty)^{d}$. If the function $g$ does not fulfill corresponding restrictions, the value function $v$ will converge to the minimal function $\hat{g}$ that is above $g$ and satisfies the restrictions.

Since the function $H$ from (3.2) describing the gamma constraint is in general not decreasing, we will have to work with the function

$$
\widehat{H}(A):=\sup _{B \in \mathcal{S}_{+}^{d}} H(A+B), \quad A \in \mathcal{S}^{d} .
$$

As in Lemma 3.3, it can be shown that $\widehat{H}$ inherits the concavity of $H$. Hence, it is either $\mathbb{R}$-valued and continuous or identically equal to $\infty$.

By $\mathcal{G}(g)$ we denote the set of all viscosity supersolutions of the equation

$$
\min \left\{f-g ; \widehat{H}\left(\operatorname{diag}[s] D^{2} f(s) \operatorname{diag}[s]\right)\right\}=0, \quad s \in(0, \infty)^{d},
$$

and we define

$$
\hat{g}(s):=\inf _{f \in \mathcal{G}(g)} f(s) .
$$

It can be made sure that $\hat{g}$ is finite by making the assumption that there exists a finite constant $c^{*}$ such that

$$
g(s) \leqslant G(s):=c^{*}\left[1+s_{1}+s_{2}+\cdots+s_{d}\right], \quad \text { for all } s \in(0, \infty)^{d} .
$$

Since by assumption (2.7), $0 \in K$, it is clear that $G \in \mathcal{G}(g)$. Therefore,

$$
\hat{g} \text { also satisfies (3.6). }
$$

Also, it follows from Perron's method (see Section 4 in [5]) that $\hat{g}$ is a viscosity solution of (3.5). Moreover, for all $t \in[0, T)$, the investment process $Y(r)=y:=\left(c^{*}, \ldots, c^{*}\right), r \in[t, T]$, is admissible, and the corresponding value process starting at $x$ is given by

$$
X_{t, s, x}^{v}(T)=x+y^{T}\left(S_{t, s}(T)-s\right)=x+G\left(S_{t, s}(T)\right)-G(s) .
$$

Hence, for $x=G(s), X_{t, s, x}^{v}(T)=G\left(S_{t, s}(T)\right) \geqslant g\left(S_{t, s}(T)\right)$, and therefore, $v(t, s) \leqslant G(s)$ for all $s \in(0, \infty)^{d}$.

To prove our main results we will need that one of the two following conditions is fulfilled:

$g$ is continuous and bounded

or

$g$ is lower semicontinuous, satisfies (3.6) and $K=\left[\Gamma_{*}, \operatorname{diag}\left[\gamma^{*}\right]\right]$, 
where $\left[\Gamma_{*}, \operatorname{diag}\left[\gamma^{*}\right]\right]$ is a bounded interval of symmetric matrices as in Example 3.1, containing the zero matrix in its interior. We require the upper bound in (3.9) to be a diagonal matrix because of the following: The upper bound on $\gamma$ induces

$$
\operatorname{diag}[s] D^{2} \hat{g}(s) \operatorname{diag}[s] \leqslant \Gamma^{*},
$$

in the viscosity sense, which implies

$$
D^{2} \hat{g}(s) \leqslant \operatorname{diag}[s]^{-1} \Gamma^{*} \operatorname{diag}[s]^{-1}
$$

in the viscosity sense. In the proof of Proposition 6.4 we will need $\Gamma^{*}$ to be of such a form that the matrix-valued function $\operatorname{diag}[s]^{-1} \Gamma^{*} \operatorname{diag}[s]^{-1}$ is the Hessian of a smooth function. This is the case if and only if $\Gamma^{*}$ is equal to a diagonal matrix $\operatorname{diag}\left[\gamma^{*}\right]$, in which case the smooth function can be taken as

$$
U(s):=-\sum_{j=1}^{d} \gamma_{j}^{*} \log s_{j} .
$$

\section{Lemma 3.5.}

(a) If $g$ satisfies (3.8), then $\hat{g}$ is also bounded.

(b) Assume (3.9). Then

$$
\hat{g}(s)=h_{\text {conc }}(s)+U(s),
$$

where $h_{\text {conc }}$ is the concave envelope of

$$
h(s)=g(s)-U(s) .
$$

Moreover, $\hat{g}$ is locally Lipschitz continuous and it is twice continuously differentiable for Lebesgue-almost every $s \in(0, \infty)^{d}$. In particular, it satisfies (3.5) Lebesgue-almost everywhere, and

$$
\operatorname{diag}[s] D^{2} \hat{g}(s) \operatorname{diag}[s] \leqslant \operatorname{diag}\left[\gamma^{*}\right] \quad \text { for Lebesgue-almost all } s \in(0, \infty)^{d} .
$$

Proof. (a) If $g$ satisfies (3.8), then every constant that dominates $g$ is a supersolution of (3.5). Hence, $\hat{g}$ is bounded.

(b) Assume that (3.9) holds. Then, set $\tilde{g}=h_{\text {conc }}+U$. We claim that $\hat{g}=\tilde{g}$. Indeed, since $h_{\text {conc }}$ is concave, it is Lebesgue-almost everywhere twice differentiable and $D^{2} h_{\text {conc }} \leqslant 0$. Therefore, $\operatorname{diag}[s] D^{2} \tilde{g} \operatorname{diag}[s] \leqslant \operatorname{diag}\left[\gamma^{*}\right]$ in the viscosity sense. Also, it is clear that $\tilde{g} \geqslant g$. Hence, by the definition of $\hat{g}$, it follows that $\tilde{g} \geqslant \hat{g}$. Since $\hat{g} \in \mathcal{G}(g)$, $D^{2} \hat{g}-\operatorname{diag}[s]^{-1} \operatorname{diag}\left[\gamma^{*}\right] \operatorname{diag}[s]^{-1} \leqslant 0$ in the viscosity sense, implying that $\hat{g}-U$ is concave. Since $\hat{g} \geqslant g$, we have $\hat{g}-U \geqslant h$. Therefore, it follows that $\hat{g}-U \geqslant h_{\text {conc }}$ and consequently, $\hat{g} \geqslant \tilde{g}$.

To prove the regularity of $\hat{g}$, observe that $h_{\text {conc }}=\hat{g}(s)-U$ is concave in $(0, \infty)^{d}$. Therefore, it is locally Lipschitz and twice differentiable Lebesgue-almost everywhere. The same holds true for $\hat{g}$. At points of twice differentiability, (3.5) holds pointwise. Hence, $\operatorname{diag}[s] D^{2} \hat{g} \operatorname{diag}[s] \leqslant \operatorname{diag}\left[\gamma^{*}\right]$ at points of twice differentiability.

\subsection{Viscosity characterization}

The chief result of this paper is the following characterization of the function $v$. In addition to characterizing $v$ as the unique viscosity solution of Eq. (3.4), we also describe the exact terminal condition satisfied by $v$. In many cases, the characterization of the terminal condition is the key to obtain an explicit solution by solving the Black-Scholes equation with this modified terminal condition.

Theorem 3.6 (Viscosity Property). Assume that (3.8) or (3.9) holds. Then, $v$ is a continuous viscosity solution of (3.4) and there exists a constant $C$ so that

$$
|v(t, s)-\hat{g}(s)| \leqslant C, \quad \text { for all }(t, s) \in[0, T) \times(0, \infty)^{d} .
$$


Theorem 3.7 (Terminal Condition). Assume that (3.8) or (3.9) holds. Then, $v$ extends to a continuous function $\hat{v}$ on $[0, T] \times[0, \infty)^{d}$ satisfying the terminal condition

$$
\hat{v}(T, s)=\hat{g}(s), \quad \text { for all } s \in(0, \infty)^{d} .
$$

In particular, $\hat{g}$ is continuous.

To prove Theorems 3.6 and 3.7 we will introduce a lower semicontinuous function $\underline{v}$ on $[0, T] \times[0, \infty)^{d}$ and an upper semicontinuous function $\bar{v}$ on $[0, T] \times[0, \infty)^{d}$ such that

$$
\underline{v} \leqslant v \leqslant \bar{v} \quad \text { on }[0, T] \times(0, \infty)^{d} .
$$

In Section 4 we show that $\bar{v}$ is a viscosity subsolution of Eq. (3.4) and in Section 5 that $\underline{v}$ is a viscosity supersolution of (3.4). In Section 6 we show that

$$
\underline{v}(T, \cdot) \geqslant \hat{g}, \quad \bar{v}(T, \cdot) \leqslant \hat{g}
$$

and there exists a constant $C>0$ such that for all $(t, s) \in[0, T] \times(0, \infty)^{d}$,

$$
\underline{v}(t, s) \geqslant \hat{g}(s)-C \quad \text { and } \quad \bar{v}(t, s) \leqslant \hat{g}(s)+C .
$$

From the comparison result, Proposition 3.9 below, we can then deduce that $\underline{v} \geqslant \bar{v}$, which implies

$$
\underline{v}=v=\bar{v}
$$

and completes the proof of Theorems 3.6 and 3.7.

The following theorem shows that $v$ is the unique solution of Eq. (3.4) in a certain class of functions.

Theorem 3.8 (Uniqueness). Assume that either (3.8) or (3.9) holds. Let w be a viscosity solution of Eq. (3.4) that satisfies the conditions (3.10),

$$
w^{*}(T, s) \leqslant \hat{g}(s)
$$

and

$$
w_{*}(T, s) \geqslant \hat{g}(s) .
$$

Then $w=v$.

Uniqueness is an immediate consequence of the following comparison result, which will be proved in Section 7.

Proposition 3.9 (Comparison). Assume that g satisfies (3.6). Suppose that $u$ is an upper semicontinuous viscosity subsolution of (3.4) and (3.12), and $w$ is a lower semicontinuous viscosity supersolution of (3.4) and (3.13). If there exists a constant $C$ so that

$$
u(t, s) \leqslant \hat{g}(s)+C \text { and } w(t, s) \geqslant \hat{g}(s)-C
$$

for all $(t, s) \in[0, T) \times(0, \infty)^{d}$, then

$$
u(t, s) \leqslant w(t, s) \quad \text { for all } \quad(t, s) \in[0, T) \times(0, \infty)^{d} .
$$

Assumption (3.14) is essentially a growth condition. It can be slightly weakened by taking $C$ to be a sublinear function of $s$. Here we chose to work with a constant to simplify the presentation. However, without an assumption of this type, comparison does not hold as illustrated in the example below. 
Example 3.10. Consider Eq. (3.4) in one dimension with $\Gamma^{*}=1, \Gamma_{*}=-\infty, \sigma(s) \equiv \sigma$ and

$$
g(s)=(s-\log s-1) \mathbf{1}_{[1, \infty)}(s) .
$$

Note that for $s>1, s^{2} g^{\prime \prime}(s)=1$. Hence, $\hat{g}=g$. Since $\Gamma_{*}=-\infty$, Eq. (3.4) has the form

$$
\min \left\{-v_{t}-\frac{1}{2} \sigma^{2} s^{2} v_{s s} ; 1-s^{2} v_{s s}\right\}=0, \quad(t, s) \in[0, T) \times(0, \infty) .
$$

Let $f$ be the function defined by $f(t)=1+(T-t)$ and set

$$
u(t, s):=g(f(t) s) .
$$

(i) First, consider the case $\sigma=0$. Then, it can easily be checked that both functions $u$ and $g$ are viscosity solutions of (3.15) and the terminal condition (3.11). Hence, comparison does not hold. Notice that Condition (3.14) is not satisfied.

(ii) For $\sigma>0$, the function $u$ is a viscosity subsolution of (3.15) and (3.11). The Black-Scholes solution

$$
w(t, s)=\mathrm{E}\left[g\left(S_{t, s}(T)\right]\right.
$$

solves (3.15) and (3.11). Moreover, clearly $w(t, s) \leqslant s$ and, for $t<T$ and sufficiently large $s$, we have $s<$ $u(t, s)$. This provides another counterexample to comparison.

Remark 3.11. Consider the Black-Scholes prices

$$
v^{\mathrm{BS}}(t, s)=\mathrm{E}\left[g\left(S_{t, s}(T)\right)\right] \quad \text { and } \hat{v}^{\mathrm{BS}}(t, s)=\mathrm{E}\left[\hat{g}\left(S_{t, s}(T)\right)\right]
$$

corresponding to $g$ and $\hat{g}$, respectively. Obviously, $v^{\mathrm{BS}} \leqslant \hat{v}^{\mathrm{BS}}$, and $v^{\mathrm{BS}}<\hat{v}^{\mathrm{BS}}$ and $v^{\mathrm{BS}}(t, s)<\hat{v}^{\mathrm{BS}}(t, s)$ if $P\left[g\left(S_{t, s}(T)\right)<\hat{g}\left(S_{t, s}(T)\right)\right]>0$. Note that $\hat{v}^{\mathrm{BS}}(T, \cdot)=\hat{g}$. Also,

$$
\mathcal{L} \hat{v}^{\mathrm{BS}}(t, s)=0,
$$

which implies

$$
\widehat{F}\left(s, \hat{v}_{t}^{\mathrm{BS}}(t, s), D^{2} \hat{v}^{\mathrm{BS}}(t, s)\right) \leqslant L\left(s, \hat{v}_{t}^{\mathrm{BS}}(t, s), D^{2} \hat{v}^{\mathrm{BS}}(t, s)\right)=0
$$

for all $(t, s) \in[0, T) \times(0, \infty)^{d}$. Hence, $\hat{v}^{\mathrm{BS}}$ is a viscosity subsolution of (3.4) and (3.12). If (3.8) or (3.9) holds, then it can also be shown that there exists a constant $C \geqslant 0$ such that

$$
\hat{v}^{\mathrm{BS}}(t, s) \leqslant \hat{g}(s)+C \quad \text { for all }(t, s) \in[0, T) \times(0, \infty)^{d} .
$$

Hence, it follows from Proposition 3.9 that

$$
\hat{v}^{\mathrm{BS}}(t, s) \leqslant v(t, s) \text { for all }(t, s) \in[0, T) \times(0, \infty)^{d} .
$$

However, if the class of trading strategies is larger than $\mathcal{A}_{t, s}$, it can happen that $v \leqslant v^{\mathrm{BS}}$ and Theorems 3.6 and 3.7 are no longer valid. For instance, it follows from Lemma A.3 of [13] that if the number of jumps $N$ in the definition (2.4) is only required to be finite but not bounded, then for every $\varepsilon>0, g\left(S_{t, s}(T)\right)$ can be super-replicated with initial capital $v^{\mathrm{BS}}(t, s)+\varepsilon$ and a strategy of the form (2.4) with $\alpha=0$ and $\gamma=0$. Theorem 4.4 of [1] shows that if $\|\alpha\|_{t, s}^{\beta, \infty}$ is not required to be finite, then for every $\varepsilon>0, g\left(S_{t, s}(T)\right)$ can be super-replicated with initial capital $v^{\mathrm{BS}}(t, s)+\varepsilon$ and a strategy of the form (2.4) without jumps and with $\gamma=0$.

\section{Viscosity subsolution property}

We here prove the subsolution property for a convenient upper semicontinuous majorant $\bar{v}$ of $v$. It will follow from the results in Sections 5, 6 and 7 that $\bar{v}=v$ if either (3.8) or (3.9) is satisfied. 


\subsection{The function $\bar{v}$}

For $v=\left(\left(\tau_{n}, y^{n}\right)_{n \geqslant 0}, \alpha, \gamma\right) \in \mathcal{A}_{t, s}$, we define

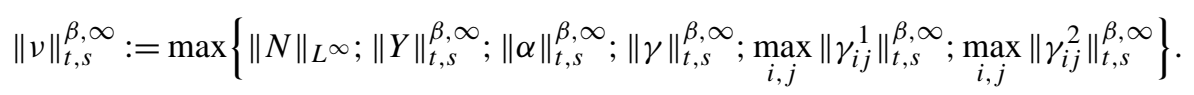

To define $\bar{v}$, we consider for every $M>0$, the set

$$
\mathcal{A}_{t, s}^{M}:=\left\{v \in \mathcal{A}_{t, s}:\|v\|_{t, s}^{\beta, \infty} \leqslant M\right\},
$$

and we introduce the associated stochastic control problem

$$
v^{M}(t, s):=\inf \left\{x \in \mathbb{R}: X_{t, s, x}^{v}(T) \geqslant g\left(S_{t, s}(T)\right) \text { for some } v \in \mathcal{A}_{t, s}^{M}\right\} .
$$

Clearly, we have

$$
\mathcal{A}_{t, s}=\bigcup_{M>0} \mathcal{A}_{t, s}^{M}, \quad \text { and therefore, } \quad v(t, s)=\inf _{M>0} v^{M}(t, s) .
$$

The function $\bar{v}$ is defined by

$$
\bar{v}(t, s):=\inf _{M>0}\left(v^{M}\right)^{*}(t, s), \quad(t, s) \in[0, T] \times[0, \infty)^{d} .
$$

Since $\bar{v}$ is an infimum of upper semicontinuous functions, it is upper semicontinuous as well, and therefore, $\bar{v} \geqslant v^{*}$.

\subsection{Partial dynamic programming principle}

Lemma 4.1. Let $t \in[0, T), s \in(0, \infty)^{d}, x \in \mathbb{R}$ and $\theta a[t, T]$-valued $\mathbb{F}^{W}$-stopping time. Let $M_{1}, M_{2}>0$ and $v \in \mathcal{A}_{t, s}^{M_{1}}$, such that

$$
X_{t, s, x}^{v}(\theta)>v^{M_{2}}\left(\theta, S_{t, s}(\theta)\right) .
$$

Then there exists a control $\hat{v} \in \mathcal{A}_{t, s}^{M_{1}+M_{2}}$ such that

$$
X_{t, s, x}^{\hat{v}}(T) \geqslant g\left(S_{t, s}(T)\right) .
$$

Proof. Set $(\varsigma, \xi):=\left(S_{t, s}(\theta), X_{t, s, x}^{v}(\theta)\right)$. It can be deduced from the definition of the control problem (4.1) and a measurable selection argument, that there exists a control $\tilde{v} \in \mathcal{A}_{\theta, 5, \xi}^{M_{2}}$ such that

$$
X_{\theta, 5, \xi}^{\tilde{v}}(T) \geqslant g\left(S_{\theta, 5}(T)\right)
$$

This step is not trivial. For more details we refer the reader to [17]. Next, let $\widehat{Y}$ be given by

$$
\widehat{Y}(r):=\mathbf{1}_{\{t \leqslant r<\theta\}} Y_{t, s}^{v}(r)+\mathbf{1}_{\{\theta \leqslant r \leqslant T\}} Y_{\theta, \zeta}^{\tilde{v}}(r)
$$

and note that the corresponding control $\hat{v}$ is in $\mathcal{A}_{t, s}^{M_{1}+M_{2}}$. It follows from (4.2) that

$$
X_{t, s, x}^{\hat{v}}(T)=X_{\theta, \zeta, \xi}^{\tilde{v}}(T) \geqslant g\left(S_{\theta, \zeta}(T)\right)=g\left(S_{t, s}(T)\right),
$$

which proves the lemma. 


\subsection{Proof of the viscosity subsolution property}

Theorem 4.2. If $g$ satisfies (3.6), then the function $\bar{v}$ is a viscosity subsolution of the equation

$$
\widehat{F}\left(s, v_{t}(t, s), D^{2} v(t, s)\right)=0 \quad \text { on }[0, T) \times(0, \infty)^{d} .
$$

Proof. By (2.8) and assumption (3.6), $0 \leqslant \bar{v}(t, s) \leqslant G(s)$ for all $(t, s) \in[0, T) \times(0, \infty)^{d}$. In particular, $\bar{v}$ is finite. Let $\left(t_{0}, s_{0}\right) \in[0, T) \times(0, \infty)^{d}$ and $\varphi \in C^{\infty}\left([0, T] \times[0, \infty)^{d}\right)$ such that

$$
0=(\bar{v}-\varphi)\left(t_{0}, s_{0}\right)>(\bar{v}-\varphi)(t, s) \text { for all }(t, s) \neq\left(t_{0}, s_{0}\right) \text {. }
$$

Assume that for some matrix $B \in \mathcal{S}_{+}^{d}$,

$$
l\left(t_{0}, s_{0}\right):=-\mathcal{L} \varphi\left(t_{0}, s_{0}\right)-\frac{1}{2} \operatorname{Tr}\left[\sigma^{T}\left(s_{0}\right) \operatorname{diag}[s] B \operatorname{diag}[s] \sigma\left(s_{0}\right)\right]>0,
$$

and

$$
h\left(t_{0}, s_{0}\right):=\operatorname{diag}\left[s_{0}\right]\left(D^{2} \varphi\left(t_{0}, s_{0}\right)+B\right) \operatorname{diag}\left[s_{0}\right] \in \operatorname{int}(K) .
$$

In the following steps we will obtain a contradiction.

1. Observe that the functions $l$ and $h$ inherit the smoothness of $\varphi$. Consider the following neighborhood of $\left(t_{0}, s_{0}\right)$ :

$$
\mathcal{N}:=\left\{(t, s) \in[0, T) \times(0, \infty)^{d} \cap B_{1}\left(t_{0}, s_{0}\right): l(t, s)>0 \text { and } h(t, s) \in \operatorname{int}(K)\right\},
$$

where $B_{1}\left(t_{0}, s_{0}\right)$ is the closed unit ball in $\mathbb{R}^{d+1}$ around $\left(t_{0}, s_{0}\right)$. Choose a constant $M_{1} \geqslant 2$ such that for each fixed pair $(\hat{t}, \hat{s}) \in \mathcal{N}$, all the functions

$$
\begin{aligned}
& D \varphi(t, s)+B(s-\hat{s}), \quad \mathcal{L} D \varphi(t, s), \quad D^{2} \varphi(t, s)+B, \quad \max _{i j}\left|\mathcal{L} D_{i j}^{2} \varphi(t, s)\right|, \\
& \max _{i j}\left|\left(D D_{i j}^{2} \varphi(t, s)\right)^{T} \operatorname{diag}[s] \sigma(s)\right|
\end{aligned}
$$

are bounded by $M_{1}$ on $\overline{\mathcal{N}}$. By definition, $\bar{v}=\inf _{M>0}\left(v^{M}\right)^{*}$. Therefore, it can be deduced from the fact that $\left(t_{0}, s_{0}\right)$ is a strict maximizer of $\bar{v}-\varphi, \partial \mathcal{N}$ is compact and $\left(v^{M}\right)^{*}-\varphi$ is upper semicontinuous for all $M$, that there exists an $\eta>0$ and an $M_{2}>0$ such that

$$
\left(v^{M_{2}}\right)^{*}(t, s) \leqslant \varphi(t, s)-4 \eta \quad \text { for all }(t, s) \in \partial \mathcal{N} .
$$

2. Let $M_{3}:=M_{1}+M_{2}$. There exists a $(\hat{t}, \hat{s}) \in \mathcal{N}$ such that

$$
v^{M_{3}}(\hat{t}, \hat{s}) \geqslant\left(v^{M_{3}}\right)^{*}\left(t_{0}, s_{0}\right)-\eta \geqslant \bar{v}\left(t_{0}, s_{0}\right)-\eta=\varphi\left(t_{0}, s_{0}\right)-\eta \geqslant \varphi(\hat{t}, \hat{s})-2 \eta .
$$

We set $\widehat{S}:=S_{\hat{t}, \hat{s}}$ and consider the stopping time

$$
\theta:=\inf \{t \geqslant \hat{t}:(t, \widehat{S}(t)) \notin \mathcal{N}\} .
$$

Then, $\theta>\hat{t}$ and $(\theta, \widehat{S}(\theta)) \in \partial \mathcal{N}$ because the process $\widehat{S}$ is almost surely continuous. Therefore,

$$
\left(v^{M_{2}}\right)^{*}(\theta, \widehat{S}(\theta)) \leqslant \varphi(\theta, \widehat{S}(\theta))-4 \eta .
$$

3. Set

$$
\begin{aligned}
& \left(\hat{\tau}_{0}, \hat{\tau}_{1}, \hat{\tau}_{2}\right)=(\hat{t}, \theta, T), \quad \hat{y}^{0}:=D \varphi(\hat{t}, \hat{s}), \quad \hat{y}^{1}:=0, \\
& \hat{\alpha}(r):=\mathbf{1}_{\{\hat{t} \leqslant r<\theta\}} \mathcal{L} D \varphi(r, \widehat{S}(r)) \quad \text { and } \quad \hat{\gamma}(r):=\mathbf{1}_{\{\hat{t} \leqslant r<\theta\}}\left(D^{2} \varphi(r, \widehat{S}(r))+B\right) .
\end{aligned}
$$

By our choice of $M_{1}$, the corresponding control $\hat{v}$ is in $\mathcal{A}_{\hat{t}, \hat{s}}^{M_{1}}$. 
4. Consider the initial capital

$$
\hat{x}:=v^{M_{3}}(\hat{t}, \hat{s})-\eta
$$

and denote $(\widehat{X}, \widehat{Y}):=\left(X_{\hat{t}, \hat{s}, \hat{x}}^{\hat{v}}, Y_{\hat{t}, \hat{s}}^{\hat{v}}\right)$. Then,

$$
\begin{aligned}
\widehat{X}(\theta)-v^{M_{2}}(\theta, \widehat{S}(\theta)) & =v^{M_{3}}(\hat{t}, \hat{s})-\eta+\int_{\hat{t}}^{\theta} \widehat{Y}(r)^{T} \mathrm{~d} \widehat{S}(r)-v^{M_{2}}(\theta, \widehat{S}(\theta)) \\
& \geqslant \varphi(\hat{t}, \hat{s})-3 \eta+\int_{\hat{t}}^{\theta} \widehat{Y}(r)^{T} \mathrm{~d} \widehat{S}(r)-v^{M_{2}}(\theta, \widehat{S}(\theta))
\end{aligned}
$$

by (4.3). Using (4.4) and Itô's lemma, we see that

$$
\begin{aligned}
\widehat{X}(\theta)-v^{M_{2}}(\theta, \widehat{S}(\theta)) & \geqslant \varphi(\hat{t}, \hat{s})-3 \eta+\int_{\hat{t}}^{\theta} \widehat{Y}(r)^{T} \mathrm{~d} \widehat{S}(r)-\varphi(\theta, \widehat{S}(\theta))+4 \eta \\
& =-\int_{\hat{t}}^{\theta} \mathcal{L} \varphi(r, \widehat{S}(r)) \mathrm{d} r+\int_{\hat{t}}^{\theta}\left(\int_{\hat{t}}^{r} B \mathrm{~d} \widehat{S}(u)\right)^{T} \mathrm{~d} \widehat{S}(r)+\eta \\
& =\int_{\hat{t}}^{\theta} l(r, \widehat{S}(r)) \mathrm{d} r+\frac{1}{2}(\widehat{S}(\theta)-\hat{s})^{T} B(\widehat{S}(\theta)-\hat{s})+\eta .
\end{aligned}
$$

Since $B \in \mathcal{S}_{+}^{d}$, this provides

$$
\widehat{X}(\theta)-v^{M_{2}}(\theta, \widehat{S}(\theta)) \geqslant \int_{\hat{t}}^{\theta} l(r, \widehat{S}(r)) \mathrm{d} r+\eta \geqslant \eta,
$$

by definition of $\theta$ as the first exit time from $\mathcal{N}$. Hence, it follows from Lemma 4.1 that $v^{M_{3}}(\hat{t}, \hat{s}) \leqslant \hat{x}$, contradicting (4.5).

\section{Viscosity supersolution property}

In this section, we prove that a convenient lower semicontinuous function $\underline{v} \leqslant v$ is a viscosity supersolution of the DPE (3.4). It will follow from results in the next sections that $\underline{v}=v$ if (3.8) or (3.9) holds.

\subsection{A weak formulation of the super-replication problem}

For technical reasons, we here also consider controls that are not necessarily adapted to the filtration generated by the Brownian motion driving the price process $S$. Let $\widetilde{W}$ be a Brownian motion on a filtered probability space $(\widetilde{\Omega}, \widetilde{\mathcal{F}}, \widetilde{\mathbb{F}}=\{\widetilde{\mathcal{F}}(t), t \in[0, T]\}, \widetilde{P})$ that satisfies the usual conditions.

For all $(t, s) \in[0, T] \times(0, \infty)^{d}$ and $M>0$, we define $\widetilde{S}_{t, s}$ and $\mathcal{A}_{t, s}^{M}(\widetilde{\Omega})$ on $(\widetilde{\Omega}, \widetilde{\mathcal{F}}, \widetilde{\mathbb{F}}, \widetilde{P})$ like $S_{t, s}$ and $\mathcal{A}_{t, s}^{M}$ are defined on $\left(\Omega, \mathcal{F}, \mathbb{F}^{W}, P\right)$. For $x \in \mathbb{R}$ and a control $\tilde{v} \in \mathcal{A}_{t, s}^{M}(\widetilde{\Omega})$, the processes $\tilde{Y}_{t, s}^{\tilde{v}}$ and $\tilde{X}_{t, s, x}^{\tilde{v}}$ are defined analogously to $Y_{t, s}^{v}$ and $X_{t, s, x}^{v}$.

Note that since $\widetilde{S}_{t, s}$ is the unique strong solution of the $\operatorname{SDE}$ (2.1) with driving Brownian motion $\widetilde{W}$, it is adapted to the filtration $\mathbb{F}^{\widetilde{W}}$ generated by $\widetilde{W}$. But, the control processes in $\mathcal{A}_{t, s}^{M}(\widetilde{\Omega})$ and therefore, $\widetilde{Y}_{t, s}^{\tilde{v}}$ and $\widetilde{X}_{t, s, x}^{\tilde{v}}$ are not necessarily adapted to $\mathbb{F}^{\widetilde{W}}$. 
For $M>0$, we define the relaxed stochastic control problem

$$
\underline{v}^{M}(t, s):=\inf \left\{x \in \mathbb{R}: \tilde{X}_{t, s, x}^{\tilde{v}}(T) \geqslant g\left(\widetilde{S}_{t, s}\right) \text { for some }(\widetilde{\Omega}, \widetilde{\mathcal{F}}, \widetilde{\mathbb{F}}, \widetilde{P}) \text { and } \tilde{v} \in \mathcal{A}_{t, s}^{M}(\widetilde{\Omega})\right\},
$$

and the functions

$$
\tilde{v}(t, s):=\inf _{M>0} \underline{v}^{M}(t, s), \quad(t, s) \in[0, T) \times(0, \infty)^{d},
$$

and

$$
\underline{v}(t, s):=\tilde{v}_{*}(t, s), \quad(t, s) \in[0, T] \times[0, \infty)^{d} .
$$

By definition, $\underline{v}$ is lower semicontinuous, and obviously, $\underline{v}(t, s) \leqslant v(t, s)$ on $[0, T) \times(0, \infty)^{d}$.

\subsection{Partial dynamic programming principle}

Lemma 5.1. Let $\widetilde{W}$ be a d-dimensional Brownian motion on a filtered probability space $(\widetilde{\Omega}, \widetilde{\mathcal{F}}, \widetilde{\mathbb{F}}=\{\widetilde{\mathcal{F}}(t)$, $t \in[0, T]\}, \widetilde{P})$ that satisfies the usual conditions. Let $(t, s) \in[0, T) \times(0, \infty)^{d}, x \in \mathbb{R}, M>0$ and $\tilde{v} \in \mathcal{A}_{t, s}^{M}(\widetilde{\Omega})$ such that

$$
\widetilde{X}_{t, s, x}^{\tilde{v}}(T) \geqslant g\left(\widetilde{S}_{t, s}(T)\right) .
$$

Then, for every $[t, T]$-valued $\widetilde{\mathbb{F}}$-stopping time $\theta$,

$$
\tilde{X}_{t, s, x}^{\tilde{v}}(\theta) \geqslant \underline{v}^{M}\left(\theta, \widetilde{S}_{t, s}(\theta)\right) .
$$

Proof. Let

$$
(\varsigma, \xi):=\left(\widetilde{S}_{t, s}, \tilde{X}_{t, s, x}^{\tilde{v}}\right)(\theta),
$$

and denote by $\mu$ the probability measure $\widetilde{P} \circ(\theta, \varsigma, \xi)^{-1}$ on $[t, T] \times \mathbb{R}^{d+1}$. Since $\tilde{X}_{t, s, x}^{\tilde{v}}(T)=\tilde{X}_{\theta, \zeta, \xi}^{\tilde{v}}(T)$ and $\widetilde{S}_{t, s}(T)=\widetilde{S}_{\theta, \zeta}(T)$, it follows from (5.1) that

$$
\begin{aligned}
1 & =\widetilde{P}\left[\widetilde{X}_{t, s, x}^{\tilde{v}}(T) \geqslant g\left(\widetilde{S}_{t, s}(T)\right)\right]=\widetilde{P}\left[\tilde{X}_{\theta, \varsigma, \xi}^{\tilde{v}}(T) \geqslant g\left(\widetilde{S}_{\theta, \zeta}(T)\right)\right] \\
& =\int_{[t, T] \times \mathbb{R}^{d+1}} \widetilde{P}\left[\widetilde{X}_{\theta, \varsigma, \xi}^{\tilde{v}}(T) \geqslant g\left(\widetilde{S}_{\theta, \zeta}(T)\right) \mid(\theta, \varsigma, \xi)=\left(t^{\prime}, s^{\prime}, x^{\prime}\right)\right] \mathrm{d} \mu\left(t^{\prime}, s^{\prime}, x^{\prime}\right) \\
& =\int_{[t, T] \times \mathbb{R}^{d+1}} \widetilde{P}\left[\tilde{X}_{t^{\prime}, s^{\prime}, x^{\prime}}^{\tilde{v}}(T) \geqslant g\left(\widetilde{S}_{t^{\prime}, s^{\prime}}(T)\right)\right] \mathrm{d} \mu\left(t^{\prime}, s^{\prime}, x^{\prime}\right) .
\end{aligned}
$$

Hence, for $\mu$-almost all $\left(t^{\prime}, s^{\prime}, x^{\prime}\right) \in[t, T] \times \mathbb{R}^{d+1}$, the control $\tilde{v}$ satisfies

$$
\widetilde{P}\left[\tilde{X}_{t^{\prime}, s^{\prime}, x^{\prime}}^{\tilde{v}}(T) \geqslant g\left(\widetilde{S}_{t^{\prime}, s^{\prime}}(T)\right)\right]=1 .
$$

Now, observe that the control corresponding to the restricted strategy $\left.\widetilde{Y}_{t, s}^{\tilde{v}}\right|_{\left[t^{\prime}, T\right]}$ belongs to $\mathcal{A}_{t^{\prime}, s^{\prime}}^{M}(\widetilde{\Omega})$. Therefore, we can conclude that for $\mu$-almost all $\left(t^{\prime}, s^{\prime}, x^{\prime}\right) \in[t, T] \times \mathbb{R}^{d+1}$,

$$
x^{\prime} \geqslant \underline{v}^{M}\left(t^{\prime}, s^{\prime}\right)
$$

which shows that $\tilde{X}_{t, s, x}^{\tilde{v}}(\theta) \geqslant \underline{v}^{M}\left(\theta, \widetilde{S}_{t, s}(\theta)\right)$. 


\subsection{Properties of $\underline{v}^{M}$}

Lemma 5.2. Assume that $g$ satisfies (3.6) for a constant $c^{*}>0$. Then, for all $M \geqslant c^{*}, \underline{v}^{M}$ is a finite, lower semicontinuous function and existence holds for the problem $\underline{v}^{M}$.

Proof. For all $M \geqslant c^{*}, \underline{v}^{M}(t, s)$ is dominated by the function $G(s)$ from (3.6). Together with (2.8), this shows that $\underline{v}^{M}$ is finite.

Next, we show that for all $M \geqslant c^{*}$, existence holds for $\underline{v}^{M}$. So, fix $M \geqslant c^{*},(t, s) \in[0, T) \times(0, \infty)^{d}$ and set

$$
x:=\underline{v}^{M}(t, s), \quad x_{k}:=x+\frac{1}{k}, \quad k \geqslant 1 .
$$

By definition of the problem $\underline{v}^{M}$, there exists for all $k \geqslant 1$, a $d$-dimensional Brownian motion $W^{k}$ on a filtered probability space $\left(\Omega^{k}, \mathcal{F}^{k}, \mathbb{F}^{k}, P^{k}\right)$ that satisfies the usual conditions and a control $v^{k} \in \mathcal{A}_{t, s}^{M}\left(\Omega^{k}\right)$ such that

$$
X_{t, s, x_{k}}^{v^{k}}(T) \geqslant g\left(S_{t, s}^{k}(T)\right) .
$$

By Lemma 5.3 below, there exists a $d$-dimensional Brownian motion $\widetilde{W}$ on a filtered probability space $(\widetilde{\Omega}, \widetilde{\mathcal{F}}, \widetilde{\mathbb{F}}, \widetilde{P})$ that satisfies the usual conditions and a control $\tilde{v} \in \mathcal{A}_{t, s}^{M}(\widetilde{\Omega})$ such that, possibly after passing to a subsequence, $\left(S_{t, s}^{k}, X_{t, s, x_{k}}^{v^{k}}\right)_{k \geqslant 1}$ converges weakly to $\left(\widetilde{S}_{t, s}, X_{t, s, x}^{\tilde{v}}\right)$. Therefore, it follows from (5.2) that

$$
\tilde{X}_{t, s, x}^{\tilde{v}}(T) \geqslant g\left(\widetilde{S}_{t, s}(T)\right) .
$$

To show that $\underline{v}$ is lower semicontinuous, we let $\left(t_{k}, s_{k}\right)_{k} \geqslant 1$ be a sequence converging to $(t, s)$, and assume that

$$
x_{k}:=\underline{v}^{M}\left(t_{k}, s_{k}\right) \rightarrow x \text { for some } x \in \mathbb{R} .
$$

Again, it can be shown that there exists a $d$-dimensional Brownian motion $\widetilde{W}$ on a filtered probability space $(\widetilde{\Omega}, \widetilde{\mathcal{F}}, \widetilde{\mathbb{F}}, \widetilde{P})$ that satisfies the usual conditions and a control $\tilde{v} \in \mathcal{A}_{t, s}^{M}(\widetilde{\Omega})$ such that

$$
\tilde{X}_{t, s, x}^{\tilde{v}}(T) \geqslant g\left(\widetilde{S}_{t, s}(T)\right) .
$$

This shows that $x \geqslant \underline{v}^{M}(t, s)$. Hence, $\underline{v}^{M}$ is lower semicontinuous.

Lemma 5.3. Let $x \geqslant 0$ and $M>0$. For every $k \geqslant 1$, let $W^{k}$ be a d-dimensional Brownian motion on a filtered probability space $\left(\Omega^{k}, \mathcal{F}^{k}, \mathbb{F}^{k}, P^{k}\right)$ that satisfies the usual conditions and $v^{k}$ a control in $\mathcal{A}_{t, s}^{M}\left(\Omega^{k}\right)$. Then there exists a d-dimensional Brownian motion $\widetilde{W}$ on a filtered probability space $(\widetilde{\Omega}, \widetilde{\mathcal{F}}, \widetilde{\mathbb{F}}, \widetilde{P})$ that satisfies the usual conditions and a control $\tilde{v} \in \mathcal{A}_{t, s}^{M}(\widetilde{\Omega})$ such that, possibly after passing to a subsequence, $\left(S_{t, s}^{k}, X_{t, s, x}^{v^{k}}\right)_{k} \geqslant 1$ converges weakly to $\left(\widetilde{S}_{t, s}, \tilde{X}_{t, s, x}^{\tilde{v}}\right)$.

Proof. For the sake of notational simplicity we assume that $d=1$. The case $d>1$ works analogously. Let $W^{\prime}$ be a one-dimensional Brownian motion on a filtered probability space $\left(\Omega^{\prime}, \mathcal{F}^{\prime}, \mathbb{F}^{\prime}, P^{\prime}\right)$ that satisfies the usual conditions and $v$ a control in $\mathcal{A}_{t, s}^{M}\left(\Omega^{\prime}\right)$. Recall that $v$ is specified by the processes

$$
\sum_{n=0}^{M-1} y^{n} \mathbf{1}_{\left[\tau_{n}, \tau_{n+1}\right)}, \quad \alpha, \quad \sum_{n=0}^{M-1} z^{n} \mathbf{1}_{\left[\tau_{n}, \tau_{n+1}\right)}, \quad \gamma^{1} \text { and } \gamma^{2} .
$$

Hence, we can identify $v$ with the process

$$
\left(\int_{t} \sum_{n=0}^{M-1} y^{n} \mathbf{1}_{\left[\tau_{n}, \tau_{n+1}\right)}(u) \mathrm{d} u, \int_{t} \alpha(u) \mathrm{d} u, \int_{t} \sum_{n=0}^{M-1} z^{n} \mathbf{1}_{\left[\tau_{n}, \tau_{n+1}\right)}(u) \mathrm{d} u, \int_{t} \gamma^{1}(u) \mathrm{d} u, \int_{t} \gamma^{2}(u) \mathrm{d} u\right)
$$


and view it as a random variable with values in $(C[t, T])^{5}$, where $C[t, T]$ denotes the space of continuous functions on $[t, T]$ endowed with the uniform topology. Then, it follows from Proposition XIII.1.5 in [14] that the sequence $\left(v^{k}\right)_{k \geqslant 1}$ is tight, and it can be shown along the lines of the proof of Theorem 7.10 in [12] that there exists a Brownian motion $\widetilde{W}$ on a filtered probability space $(\widetilde{\Omega}, \widetilde{\mathcal{F}}, \widetilde{\mathbb{F}}, \widetilde{P})$ that satisfies the usual conditions and a control $\tilde{\nu} \in \mathcal{A}_{t, s}^{M}(\widetilde{\Omega})$ such that, possibly after passing to a subsequence, $\left(v^{k}, S_{t, s}^{k}, X_{t, s, x}^{v_{k}}\right)_{k \geqslant 1}$ converges weakly to $\left(\tilde{v}, \widetilde{S}_{t, s}, \widetilde{X}_{t, s, x}^{\tilde{v}}\right)$.

\subsection{Proof of the viscosity supersolution property}

Theorem 5.4. Assume that $g$ satisfies (3.6) for a constant $c^{*}>0$. Then for all $M \geqslant c^{*}, \underline{v}^{M}$ is a viscosity supersolution of the equation

$$
\widehat{F}\left(s, v_{t}(t, s), D^{2} v(t, s)\right)=0 \quad \text { on }[0, T) \times(0, \infty)^{d} .
$$

Proof. Fix an $M \geqslant c^{*}$. By Lemma 5.2, $\underline{v}^{M}$ is finite and lower semicontinuous for all $M \geqslant c^{*}$. Consider a $\left(t_{0}, s_{0}\right) \in$ $[0, T) \times(0, \infty)^{d}$ together with a test function $\varphi \in C^{\infty}\left([0, T] \times[0, \infty)^{d}\right)$ such that

$$
0=\left(\underline{v}^{M}-\varphi\right)\left(t_{0}, s_{0}\right)=\min _{(t, s) \in[0, T] \times[0, \infty)^{d}}\left(\underline{v}^{M}-\varphi\right)(t, s) .
$$

The proof is complete if we can show that there exists a $B \in \mathcal{S}_{+}^{d}$ such that

$$
-\mathcal{L} \varphi\left(t_{0}, s_{0}\right)-\frac{1}{2} \operatorname{Tr}\left[\sigma^{T}\left(s_{0}\right) \operatorname{diag}\left[s_{0}\right] B \operatorname{diag}\left[s_{0}\right] \sigma\left(s_{0}\right)\right] \geqslant 0,
$$

and

$$
\operatorname{diag}\left[s_{0}\right]\left(D^{2} \varphi\left(t_{0}, s_{0}\right)+B\right) \operatorname{diag}\left[s_{0}\right] \in K .
$$

1. Set $x_{0}:=\underline{v}^{M}\left(t_{0}, s_{0}\right)$. By Lemma 5.2, there exists a $d$-dimensional Brownian motion $\widetilde{W}$ on a filtered probability space $(\widetilde{\Omega}, \widetilde{\mathcal{F}}, \widetilde{\mathbb{F}}, \widetilde{P})$ satisfying the usual conditions and a control $\tilde{v} \in \mathcal{A}_{t_{0}, s_{0}}^{M}(\widetilde{\Omega})$ such that

$$
\tilde{X}_{t_{0}, s_{0}, x_{0}}^{\tilde{v}}(T) \geqslant g\left(\widetilde{S}_{t_{0}, s_{0}}(T)\right) \text {. }
$$

For the rest of the proof we denote

$$
(\widetilde{S}, \tilde{Y}, \tilde{X})=\left(\widetilde{S}_{t_{0}, s_{0}}, \tilde{Y}_{t_{0}, s_{0}}^{\tilde{v}}, \tilde{X}_{t_{0}, s_{0}, x_{0}}^{\tilde{v}}\right) .
$$

Let $\tilde{\tau}_{1}$ be the first jump time after $t_{0}$ appearing in the control $\tilde{v}$ and define

$$
\theta:=\tilde{\tau}_{1} \wedge \inf \left\{r>t_{0}: \widetilde{S}(r) \notin s_{0} \mathrm{e}^{B_{1}(0)}\right\},
$$

where $B_{1}(0)$ is the closed unit ball in $\mathbb{R}^{d}$ around 0 and the exponential and product are taken component-wise. For all $\eta>0$, set

$$
\theta^{\eta}:=\theta \wedge\left(t_{0}+\eta\right),
$$

and notice that $\theta^{\eta}>t_{0} \widetilde{P}$-almost surely. By the partial dynamic programming principle of Lemma 5.1,

$$
\widetilde{X}\left(\theta^{\eta}\right) \geqslant \underline{v}^{M}\left(\theta^{\eta}, \widetilde{S}\left(\theta^{\eta}\right)\right)
$$

Since $\underline{v}^{M} \geqslant \varphi$, it follows from (5.3) that

$$
x_{0}+\int_{t_{0}}^{\theta^{\eta}} \tilde{Y}(r)^{T} \widetilde{S}(r)-\varphi\left(\theta^{\eta}, \widetilde{S}\left(\theta^{\eta}\right)\right) \geqslant 0 .
$$

By twice applying Itô's lemma, it follows that 


$$
\int_{t_{0}}^{\theta^{\eta}} l(r) \mathrm{d} r+\int_{t_{0}}^{\theta^{\eta}}\left(c+\int_{t_{0}}^{r} a(u) \mathrm{d} u+\int_{t_{0}}^{r} b(u) \mathrm{d} \widetilde{S}(u)\right)^{T} \mathrm{~d} \widetilde{S}(r) \geqslant 0,
$$

where

$$
\begin{aligned}
& l(r):=-\mathcal{L} \varphi(r \wedge \theta, \widetilde{S}(r \wedge \theta)), \\
& a(r):=\tilde{\alpha}(r \wedge \theta)-\mathcal{L}(D \varphi)(r \wedge \theta, \widetilde{S}(r \wedge \theta)), \\
& b(r):=\tilde{\gamma}(r \wedge \theta)-D^{2} \varphi(r \wedge \theta, \widetilde{S}(r \wedge \theta))
\end{aligned}
$$

and

$$
c:=\tilde{y}^{0}-D \varphi\left(t_{0}, s_{0}\right) .
$$

Note that, by our choice of the stopping time $\theta$, the processes $l, a$ and $b$ are bounded. Hence, there exists a constant $C_{1}>0$ such that for all $\eta>0$,

$$
\left|\int_{t_{0}}^{\theta^{\eta}} l(r) \mathrm{d} r\right| \leqslant C_{1} \eta
$$

Moreover, the process

$$
m(r):=\mathbf{1}_{\{r<\theta\}} \operatorname{diag}[\widetilde{S}(r)] \sigma(\widetilde{S}(r)), \quad r \in\left[t_{0}, T\right],
$$

satisfies the continuity assumption (A.3) of Proposition A.3 at $t_{0}$ for $\varepsilon=0$. Therefore, it follows from Proposition A.3 that for every constant $\varepsilon>0$, almost surely,

$$
\lim _{\eta \searrow 0} \eta^{-3 / 2+\varepsilon} \int_{t_{0}}^{\theta^{\eta}}\left(\int_{t_{0}}^{r} a(u) \mathrm{d} u\right)^{T} \mathrm{~d} \widetilde{S}(r)=\lim _{\eta \searrow 0} \eta^{-3 / 2+\varepsilon} \int_{t_{0}}^{t_{0}+\eta}\left(\int_{t_{0}}^{r} a(u) \mathrm{d} u\right)^{T} m(r) \mathrm{d} \widetilde{W}(r)=0 .
$$

It can easily be checked that $\widetilde{S}$ is almost surely Hölder-continuous of order $1 / 3$. Hence, the process $m$ satisfies the continuity assumption (A.1) of Theorem A.1 for $\varepsilon=2 / 3$, and it follows from Theorem A.1.a that there exists a constant $C_{2}>0$ such that

$$
\begin{aligned}
& \underset{\eta \searrow 0}{\limsup } \frac{1}{\eta \log \log (1 / \eta)}\left|\int_{t_{0}}^{\theta^{\eta}}\left(\int_{t_{0}}^{r} b(u) \mathrm{d} \widetilde{S}(u)\right)^{T} \mathrm{~d} \widetilde{S}(r)\right| \\
& \quad=\limsup _{\eta \searrow 0} \frac{1}{\eta \log \log (1 / \eta)}\left|\int_{t_{0}}^{t_{0}+\eta}\left(\int_{t_{0}}^{r} b(u) m(u) \mathrm{d} \widetilde{W}(u)\right)^{T} m(r) \mathrm{d} \widetilde{W}(r)\right| \leqslant C_{2} .
\end{aligned}
$$

2. By the Dambis-Dubins-Schwarz Theorem (see e.g. Theorem V.I.6 in [14]), there exists a Brownian motion $Z$ such that

$$
\int_{t_{0}}^{\theta_{\eta}} c^{T} \mathrm{~d} \widetilde{S}(r)=\int_{t_{0}}^{\theta_{\eta}} c^{T} m(r) \mathrm{d} \widetilde{W}(r)=Z\left(\int_{t_{0}}^{\theta_{\eta}}\left|c^{T} m(r)\right|^{2} \mathrm{~d} r\right) .
$$

Hence, it follows from (5.4)-(5.7) and the law of the iterated logarithm for Brownian motion (see e.g. Theorem II.1.9 in [14]) that $c^{T} m\left(t_{0}\right)=0$, which by our assumption that $\sigma\left(s_{0}\right)$ is invertible, implies

$$
c=0 \text {. }
$$


3. By (5.8), we can rewrite (5.4) as

$$
\int_{t_{0}}^{\theta^{\eta}} l(r) \mathrm{d} r+\int_{t_{0}}^{\theta^{\eta}}\left(\int_{t_{0}}^{r} a(u) \mathrm{d} u+\int_{t_{0}}^{r} b(u) \mathrm{d} \widetilde{S}(u)\right)^{T} \mathrm{~d} \widetilde{S}(r) \geqslant 0 .
$$

It follows from (5.9), (5.5) and (5.6) that

$$
\liminf _{\eta \searrow 0} \frac{1}{\eta \log \log (1 / \eta)} \int_{t_{0}}^{\theta^{\eta}}\left(\int_{t_{0}}^{r} b(u) \mathrm{d} \widetilde{S}(u)\right)^{T} \mathrm{~d} \widetilde{S}(r) \geqslant 0 .
$$

Since $b$ is right-continuous, it follows from (5.10) and Theorem A.1.b that

$$
m\left(t_{0}\right)^{T} b\left(t_{0}\right) m\left(t_{0}\right) \in \mathcal{S}_{+}^{d} .
$$

Since $\sigma\left(s_{0}\right)$ is invertible, this implies that $b\left(t_{0}\right) \in \mathcal{S}_{+}^{d}$, and it follows from the gamma constraint (2.6) that

$$
\operatorname{diag}\left[s_{0}\right]\left(D^{2} \varphi\left(t_{0}, s_{0}\right)+b\left(t_{0}\right)\right) \operatorname{diag}\left[s_{0}\right] \in K .
$$

By the boundedness and continuity of the process $l$, we obtain from (5.9) and (5.6) that

$$
-\mathcal{L} \varphi\left(t_{0}, s_{0}\right)=l\left(t_{0}\right) \geqslant \limsup _{\eta \searrow 0} \frac{1}{\eta} \int_{t_{0}}^{\theta^{\eta}}\left(\int_{t_{0}}^{r}-b(u) \mathrm{d} \widetilde{S}(u)\right)^{T} \mathrm{~d} \widetilde{S}(r) .
$$

Since $b$ is of the form (2.5), it satisfies the continuity assumption (A.2) of Theorem A.2. Hence, we get from (5.11) and Theorem A.2 that

$$
\begin{aligned}
& \underset{\eta \searrow 0}{\limsup } \frac{1}{\eta} \int_{t_{0}}^{\theta^{\eta}}\left(\int_{t_{0}}^{r}-b(u) \mathrm{d} \widetilde{S}(u)\right)^{T} \mathrm{~d} \widetilde{S}(r) \\
& \quad=\underset{\eta \searrow 0}{\limsup } \frac{1}{\eta} \int_{t_{0}}^{t_{0}+\eta}\left(\int_{t_{0}}^{r}-b(u) m(u) \mathrm{d} \widetilde{W}(u)\right)^{T} m(r) \mathrm{d} \tilde{W}(r) \\
& \quad=\frac{1}{2} \operatorname{Tr}\left[m\left(t_{0}\right)^{T} b\left(t_{0}\right) m\left(t_{0}\right)\right]=\frac{1}{2} \operatorname{Tr}\left[\sigma\left(s_{0}\right)^{T} \operatorname{diag}\left[s_{0}\right] b\left(t_{0}\right) \operatorname{diag}\left[s_{0}\right] \sigma\left(s_{0}\right)\right] .
\end{aligned}
$$

Together with (5.12), this shows that

$$
-\mathcal{L} \varphi\left(t_{0}, s_{0}\right)-\frac{1}{2} \operatorname{Tr}\left[\sigma\left(s_{0}\right)^{T} \operatorname{diag}\left[s_{0}\right] b\left(t_{0}\right) \operatorname{diag}\left[s_{0}\right] \sigma\left(s_{0}\right)\right] \geqslant 0,
$$

which completes the proof.

Corollary 5.5. If $g$ satisfies (3.6), then $\underline{v}$ is a viscosity supersolution of the equation

$$
\widehat{F}\left(s, v_{t}(t, s), D^{2} v(t, s)\right)=0 \quad \text { on }[0, T) \times(0, \infty)^{d} .
$$

Proof. The corollary can be deduced from Theorem 5.4 with the following argument borrowed from Remark 6.3 of [5], see also Proposition 2.3 of [19].

Let $\left(t_{0}, s_{0}\right) \in[0, T) \times(0, \infty)^{d}$ and $\varphi \in C^{\infty}\left([0, T] \times[0, \infty)^{d}\right)$ such that

$$
0=(\underline{v}-\varphi)\left(t_{0}, s_{0}\right)<(\underline{v}-\varphi)(t, s) \quad \text { for all }(t, s) \neq\left(t_{0}, s_{0}\right) \text {. }
$$


Let $B_{1}(0)$ be the closed unit ball in $\mathbb{R}^{d}$ and denote

$$
Q:=\left[0, \frac{t_{0}+T}{2}\right] \times s_{0} \mathrm{e}^{B_{1}(0)},
$$

where the product and exponential are take component-wise. By definition of $\underline{v}$, there exists a sequence $\left(t_{k}, s_{k}\right)_{k} \geqslant 1$ in $Q$ such that

$$
\left(t_{k}, s_{k}\right) \rightarrow\left(t_{0}, s_{0}\right)
$$

and

$$
\underline{v}^{k}\left(t_{k}, s_{k}\right) \rightarrow \underline{v}\left(t_{0}, s_{0}\right) .
$$

For all $k \geqslant 1$, let $\left(\underline{t}_{k}, \underline{s}_{k}\right)$ be a minimizer of $\underline{v}^{k}-\varphi$ on $Q$. Then, necessarily,

$$
\left(\underline{t}_{k}, \underline{s}_{k}\right) \rightarrow\left(t_{0}, s_{0}\right) \text {. }
$$

Indeed, assume that there exists a subsequence $\left(\underline{t}_{k_{j}}, \underline{s}_{k_{j}}\right)_{j} \geqslant 1$ of $\left(\underline{t}_{k}, \underline{s}_{k}\right)_{k} \geqslant 1$ that converges to a point $(\underline{t}, \underline{s}) \in Q$. Then,

$$
0=(\underline{v}-\varphi)\left(t_{0}, s_{0}\right)=\lim _{j \rightarrow \infty}\left(\underline{v}^{k_{j}}-\varphi\right)\left(t_{k_{j}}, s_{k_{j}}\right) \geqslant \liminf _{j \rightarrow \infty}\left(\underline{v}^{k_{j}}-\varphi\right)\left(\underline{t}_{k_{j}}, \underline{s}_{k_{j}}\right) \geqslant(\underline{v}-\varphi)(\underline{t}, \underline{s}) .
$$

Hence, $(\underline{t}, \underline{s})=\left(t_{0}, s_{0}\right)$, and (5.13) follows. By (5.13), there exists a $k_{0} \geqslant 1$ such that $\left(\underline{t}_{k}, \underline{s}_{k}\right)$ is a local minimizer of $\underline{v}^{k}-\varphi$ for all $k \geqslant k_{0}$. Hence, Theorem 5.4 implies that

$$
\widehat{F}\left(\underline{s}_{k}, \varphi_{t}\left(\underline{t}_{k}, \underline{s}_{k}\right), D^{2} \varphi\left(\underline{t}_{k}, \underline{s}_{k}\right)\right) \geqslant 0, \quad \text { for all } k \geqslant k_{0} .
$$

By Lemma 3.4, $\widehat{F}$ is continuous. Therefore, by sending $k$ to $\infty$, we get

$$
\widehat{F}\left(s_{0}, \varphi_{t}\left(t_{0}, s_{0}\right), D^{2} \varphi\left(t_{0}, s_{0}\right)\right) \geqslant 0,
$$

which proves the corollary.

\section{Terminal condition}

In the previous sections, we proved that if $g$ satisfies (3.6), then $\bar{v}$ is a viscosity subsolution and $\underline{v}$ a viscosity supersolution of Eq. (3.4). Here, our objective is to show that if one of the conditions (3.8) or (3.9) holds, then

$$
\underline{V}:=\underline{v}(T, \cdot) \quad \text { and } \quad \bar{V}:=\bar{v}(T, \cdot)
$$

satisfy the terminal condition

$$
\underline{V}=\bar{V}=\hat{g},
$$

and there exists a constant $C$ such that for all $(t, s) \in[0, T) \times(0, \infty)^{d}$,

$$
\underline{v}(t, s) \geqslant \hat{g}(s)-C \quad \text { and } \quad \bar{v}(t, s) \leqslant \hat{g}(s)+C .
$$

We first prove the lower bound $\underline{V} \geqslant \hat{g}$ and then the upper bound $\bar{V} \leqslant \hat{g}$. The lower bound can be proved under (3.6). For the proof of the upper bound we need that either (3.8) or (3.9) is satisfied, and the proof is different in the two cases. The inequalities (6.2) are trivial under (3.8) and can be deduced more or less directly from (6.1) under (3.9). 


\subsection{Lower bound under (3.6)}

Proposition 6.1. If $g$ is lower semicontinuous and satisfies (3.6), then $\underline{V}$ is a viscosity supersolution of (3.5). In particular, $\underline{V} \geqslant \hat{g}$.

Proof. 1. Let $s_{0} \in(0, \infty)$. Assumption (3.6) insures that

$$
\underline{v}(t, s) \leqslant \tilde{v}(t, s) \leqslant v(t, s) \leqslant G(s) \text { for all }(t, s) \in[0, T) \times(0, \infty)^{d},
$$

and by the definition of $\underline{v}$, there exists a sequence $\left(t_{k}, s_{k}\right)_{k \geqslant 1}$ in $[0, T) \times(0, \infty)^{d}$ converging to $\left(T, s_{0}\right)$ such that

$$
\tilde{v}\left(t_{k}, s_{k}\right) \rightarrow \underline{v}\left(T, s_{0}\right)=\underline{V}\left(s_{0}\right) .
$$

There exists for all $k \geqslant 1$, a filtered probability space $\left(\Omega^{k}, \mathcal{F}^{k}, \mathbb{F}^{k}, P^{k}\right)$ satisfying the usual conditions with a $d$-dimensional Brownian motion $W^{k}$ and a control $\nu^{k} \in \bigcup_{M>0} \mathcal{A}_{t, s}^{M}\left(\Omega^{k}\right)$ such that

$$
X_{t_{k}, s_{k}, x_{k}}^{v^{k}}(T) \geqslant g\left(S_{t_{k}, s_{k}}^{k}(T)\right),
$$

where $x_{k}:=\tilde{v}\left(t_{k}, s_{k}\right)+1 / k$. By $(2.8)$,

$$
x_{k}=\mathrm{E}^{k}\left[X_{t_{k}, s_{k}, x_{k}}^{v^{k}}(T)\right] \geqslant \mathrm{E}^{k}\left[g\left(S_{t_{k}, s_{k}}^{k}(T)\right)\right]=\mathrm{E}\left[g\left(S_{t_{k}, s_{k}}(T)\right)\right],
$$

and obviously,

$$
S_{t_{k}, s_{k}}(T) \rightarrow s_{0} \quad \text { almost surely. }
$$

Since, $g$ is lower semicontinuous, it follows from (6.3) that

$$
\liminf _{k \rightarrow \infty} g\left(S_{t_{k}, s_{k}}(T)\right) \geqslant g\left(s_{0}\right) .
$$

Therefore, Fatou's lemma implies

$$
\underline{V}\left(s_{0}\right)=\lim _{k \rightarrow \infty} x_{k} \geqslant \liminf _{k \rightarrow \infty} \mathrm{E}\left[g\left(S_{t_{k}, s_{k}}(T)\right)\right] \geqslant g\left(s_{0}\right) .
$$

2. Let $\left(s_{0}, \psi\right) \in(0, \infty)^{d} \times C[0, \infty)^{d}$ be such that

$$
0=(\underline{V}-\psi)\left(s_{0}\right)=\min _{s \in[0, \infty)^{d}}(\underline{V}-\psi)(s) .
$$

Choose a sequence $\left(t_{k}, s_{k}\right)_{k} \geqslant 1$ which converges to $\left(T, s_{0}\right)$, such that $t_{k}<T$ and

$$
\lim _{k \rightarrow \infty} \tilde{v}\left(t_{k}, s_{k}\right)=\underline{V}\left(s_{0}\right) .
$$

For all $k \geqslant 1$, we define the function $w_{k}$ on $\left[t_{k}, T\right] \times[0, \infty)^{d}$ as the lower semicontinuous envelope of the function $\tilde{v}$ restricted to $\left[t_{k}, T\right) \times(0, \infty)^{d}$. Then $w_{k}=\tilde{v}_{*}=\underline{v}$ on $\left(t_{k}, T\right] \times[0, \infty)^{d}$ but we do not know whether $w_{k}$ is equal to $\underline{v}$ on $t_{k} \times[0, \infty)^{d}$. However, we can replace 0 by $t_{k}$ in Corollary 5.5 and deduce that $w_{k}$ is a viscosity supersolution of Eq. (3.4) on $\left[t_{k}, T\right) \times(0, \infty)^{d}$. Define the auxiliary test function

$$
\varphi^{k}(t, s):=\psi(s)-\left|s-s_{0}\right|^{4}+\frac{T-t}{T-t_{k}},
$$

and denote by $B_{1}(0)$ the closed unit ball in $\mathbb{R}^{d}$ around 0 . For all $k \geqslant 1$, let $\left(\underline{t}_{k}, \underline{s}_{k}\right)$ be a minimizer of $w_{k}-\varphi^{k}$ on $\left[t_{k}, T\right] \times s_{0} \mathrm{e}^{B_{1}(0)}$. Note that

$$
\left(w_{k}-\varphi^{k}\right)\left(t_{k}, s_{k}\right)=\left(w_{k}-\psi\right)\left(t_{k}, s_{k}\right)+\left|s_{k}-s_{0}\right|^{4}-1 \rightarrow-1 \quad \text { as } k \rightarrow \infty .
$$

Hence, for $k$ large enough,

$$
\left(w_{k}-\varphi^{k}\right)\left(t_{k}, s_{k}\right)<0 .
$$


On the other hand,

$$
\left(w_{k}-\varphi^{k}\right)(T, s)=\underline{V}(s)-\psi(s)+\left|s-s_{0}\right|^{4} \geqslant 0 \quad \text { for all } s \in(0, \infty)^{d} .
$$

Hence, $\underline{t}_{k} \in\left[t_{k}, T\right)$ for large enough $k$. Now, let $s^{*} \in(0, \infty)^{d}$ such that, possibly after passing to a subsequence, $\underline{s}_{k} \rightarrow s^{*}$. Then,

$$
\begin{aligned}
\left|s^{*}-s_{0}\right|^{4} & =\lim _{k \rightarrow \infty}\left|\underline{s}_{k}-s_{0}\right|^{4} \\
& \leqslant \liminf _{k \rightarrow \infty}\left\{\left(w_{k}-\varphi^{k}\right)\left(t_{k}, s_{k}\right)-\left(w_{k}-\varphi^{k}\right)\left(\underline{t}_{k}, \underline{s}_{k}\right)+\left|\underline{s}_{k}-s_{0}\right|^{4}\right\} \\
& =\liminf _{k \rightarrow \infty}\left\{\left(w_{k}-\psi\right)\left(t_{k}, s_{k}\right)-\left(w_{k}-\psi\right)\left(\underline{t}_{k}, \underline{s}_{k}\right)+\frac{T-\underline{t}_{k}}{T-t_{k}}+\left|s_{k}-s_{0}\right|^{4}-\frac{T-t_{k}}{T-t_{k}}\right\} \\
& \leqslant \liminf _{k \rightarrow \infty}\left\{(\underline{V}-\psi)\left(s_{0}\right)-(\underline{V}-\psi)\left(s^{*}\right)-\frac{\underline{t}_{k}-t_{k}}{T-t_{k}}\right\} \leqslant 0 .
\end{aligned}
$$

This shows that $\left(\underline{t}_{k}, \underline{s}_{k}\right) \rightarrow\left(T, s_{0}\right)$ as $k \rightarrow \infty$. Since $w_{k}$ is a viscosity supersolution of $\widehat{F}\left(s, v_{t}, D^{2} v\right)=0$ on $\left[t_{k}, T\right) \times(0, \infty)^{d}$, we have

$$
\widehat{F}\left(\underline{s}_{k}, \varphi_{t}^{k}\left(\underline{t}_{k}, \underline{s}_{k}\right), D^{2} \varphi^{k}\left(\underline{t}_{k}, \underline{s}_{k}\right)\right) \geqslant 0,
$$

and in particular,

$$
\widehat{H}\left(\operatorname{diag}\left[\underline{s}_{k}\right]\left(D^{2} \psi\left(\underline{s}_{k}\right)-D^{2}\left|\underline{s}_{k}-s_{0}\right|^{4}\right) \operatorname{diag}\left[\underline{s}_{k}\right]\right) \geqslant 0,
$$

which, by continuity of $\widehat{H}$, implies

$$
\widehat{H}\left(\operatorname{diag}\left[s_{0}\right] D^{2} \psi\left(s_{0}\right) \operatorname{diag}\left[s_{0}\right]\right) \geqslant 0 .
$$

Hence, $\underline{V}$ is a viscosity supersolution of (3.5), and therefore, $\underline{V} \geqslant \hat{g}$.

The next result provides more detailed information about the lower bound if the convex set $K$ is bounded from below by a matrix of the form $-a^{*} I_{d}$, where $a^{*}$ is a constant and $I_{d}$ the $d \times d$-identity matrix.

Corollary 6.2. Assume $g$ satisfies (3.6) and for some constant $a^{*}, A \geqslant-a^{*} I_{d}$ for every $A \in K$. Then, there exists a constant $C$ such that

$$
\underline{v}(t, s) \geqslant \hat{g}(s)-C(T-t) \quad \text { on }[0, T) \times(0, \infty)^{d} .
$$

Proof. In view of the previous lemma, it suffices to show that there exists a constant $C$ so that $\underline{v}$ is a viscosity supersolution of the equation

$$
-v_{t}(t, s)+C=0 .
$$

To prove (6.4), consider $\left(t_{0}, s_{0}\right) \in[0, T) \times(0, \infty)^{d}$ and a test function $\varphi \in C^{\infty}\left([0, T] \times[0, \infty)^{d}\right)$ so that

$$
0=(\underline{v}-\varphi)\left(t_{0}, s_{0}\right)=\min _{(t, s) \in[0, T] \times[0, \infty)^{d}}(\underline{v}-\varphi)(t, s) .
$$

By Corollary 5.5, we have

$$
\widehat{F}\left(\left(t_{0}, s_{0}\right), \varphi_{t}\left(t_{0}, s_{0}\right), D^{2} \varphi\left(t_{0}, s_{0}\right)\right) \geqslant 0,
$$

which implies that there exists a $B \in \mathcal{S}_{+}^{d}$ such that

$$
-\varphi_{t}\left(t_{0}, s_{0}\right)-\frac{1}{2} \operatorname{Tr}\left[\sigma\left(s_{0}\right)^{T} \operatorname{diag}\left[s_{0}\right]\left(D^{2} \varphi\left(t_{0}, s_{0}\right)+B\right) \operatorname{diag}\left[s_{0}\right] \sigma\left(s_{0}\right)\right] \geqslant-1
$$


and

$$
H\left(\operatorname{diag}\left[s_{0}\right]\left(D^{2} \varphi\left(t_{0}, s_{0}\right)+B\right) \operatorname{diag}\left[s_{0}\right]\right) \geqslant-1 .
$$

It follows from the assumption and (6.6) that

$$
\operatorname{diag}\left[s_{0}\right]\left(D^{2} \varphi\left(t_{0}, s_{0}\right)+B\right) \operatorname{diag}\left[s_{0}\right] \geqslant-\left(a^{*}+1\right) I_{d} .
$$

Hence, by (6.5),

$$
-\varphi_{t}\left(t_{0}, s_{0}\right) \geqslant-1-\frac{1}{2}\left(a^{*}+1\right) \operatorname{Tr}\left[\sigma\left(s_{0}\right)^{T} \sigma\left(s_{0}\right)\right] .
$$

Since $\sigma$ is bounded, this implies that

$$
-\varphi_{t}\left(t_{0}, s_{0}\right) \geqslant-C
$$

for some positive constant $C$ that is independent of $\left(t_{0}, s_{0}\right)$.

Note that the hypothesis of the previous corollary holds under the assumption (3.9). Under the assumption (3.8), $\underline{v}(t, s)$ and $\hat{g}$ are bounded.

\subsection{Upper bound under (3.8)}

Note that in the proof of the following proposition we need the continuity of $g$.

Proposition 6.3. Assume (3.8). Then, $\bar{V} \leqslant \hat{g}$.

Proof. We will show that if $g$ satisfies (3.8), then $\bar{V}$ is a viscosity subsolution of (3.5). On the other hand, $\hat{g}$ is a viscosity supersolution of the same equation. Moreover, we assume that $g$ is bounded. Therefore, $\bar{V}-\hat{g}$ is bounded, and we can apply a comparison result for Eq. (3.5), to conclude that $\bar{V} \leqslant \hat{g}$ (see Remark 7.6). In the remainder of this proof, we show that $\bar{V}$ is a viscosity subsolution of (3.5).

Consider a pair $\left(s_{0}, \psi\right) \in(0, \infty)^{d} \times C^{\infty}\left([0, \infty)^{d}\right)$ such that

$$
0=(\bar{V}-\psi)\left(s_{0}\right)=\max _{[0, \infty)^{d}}(\bar{V}-\psi)
$$

and

$$
\psi\left(s_{0}\right)>g\left(s_{0}\right) .
$$

For $k \geqslant 1$, set

$$
\varphi^{k}(t, s):=\psi(s)+\left|s-s_{0}\right|^{4}+k(T-t), \quad(t, s) \in[0, T] \times[0, \infty)^{d} .
$$

Fix $B \in \mathcal{S}_{+}^{d}$ and assume that there exists a $k \geqslant 1$ such that

$$
F\left(s, \varphi_{t}^{k}(t, s), D^{2} \varphi^{k}(t, s)+B\right)>0
$$

on the set

$$
Q_{1 / k}:=[T-1 / k, T] \times s_{0} \mathrm{e}^{B_{1 / k}(0)},
$$

where $B_{1 / k}(0)$ is the closed ball of radius $1 / k$ around 0 in $\mathbb{R}^{d}$ and the product and exponential are taken componentwise. Then,

$$
-\mathcal{L} \varphi^{k}(t, s)-\frac{1}{2} \operatorname{Tr}\left[\sigma(s)^{T} \operatorname{diag}[s] B \operatorname{diag}[s] \sigma(s)\right]>0
$$


and

$$
H\left(\operatorname{diag}[s]\left(D^{2} \varphi^{k}(s)+B\right) \operatorname{diag}[s]\right)>0,
$$

for all $(t, s) \in Q_{1 / k}$. In the following steps we derive a contradiction to (6.8).

1. There exists a constant $M_{1} \geqslant 2$ such that for each fixed pair $(\hat{t}, \hat{s}) \in Q_{1 / k}$ all the functions

$$
\begin{aligned}
& D \varphi^{k}(t, s)+B(s-\hat{s}), \quad \mathcal{L} D \varphi^{k}(t, s), \quad D^{2} \varphi^{k}(t, s)+B, \quad \max _{i j}\left|\mathcal{L} D_{i j}^{2} \varphi^{k}(t, s)\right|, \\
& \max _{i j}\left|\left(D D_{i j}^{2} \varphi^{k}(t, s)\right)^{T} \operatorname{diag}[s] \sigma(s)\right|
\end{aligned}
$$

are bounded by $M_{1}$ on $Q_{1 / k}$.

2. Since $g$ is continuous, it can be deduced from $\bar{v}=\inf _{M>0}\left(v^{M}\right)^{*}$ and (6.7) that there exist $\eta \in(0,1 / k]$ and $M_{2}>0$ such that

$$
\left(v^{M_{2}}\right)^{*} \leqslant \varphi^{k}-4 \eta
$$

on the parabolic boundary

$$
\partial_{p} Q_{\eta}:=\left([T-\eta, T] \times \partial\left(s_{0} \mathrm{e}^{B_{\eta}(0)}\right) \cup\left(\{T\} \times s_{0} \mathrm{e}^{B_{\eta}(0)}\right)\right)
$$

of the set $Q_{\eta}=[T-\eta, T] \times s_{0} \mathrm{e}^{B_{\eta}(0)}$.

3. Set $M_{3}:=M_{1}+M_{2}$. There exists a $(\hat{t}, \hat{s}) \in Q_{\eta}$ such that

$$
v^{M_{3}}(\hat{t}, \hat{s}) \geqslant\left(v^{M_{3}}\right)^{*}\left(T, s_{0}\right)-\eta \geqslant \bar{v}\left(T, s_{0}\right)-\eta=\varphi^{k}\left(T, s_{0}\right)-\eta \geqslant \varphi^{k}(\hat{t}, \hat{s})-2 \eta .
$$

Denote $\widehat{S}:=S_{\hat{t}, \hat{s}}$ and introduce the stopping time

$$
\theta:=\inf \left\{r \geqslant \hat{t} \mid \widehat{S}(r) \in \partial_{p} Q_{\eta}\right\} .
$$

Then, $(\theta, \widehat{S}(\theta)) \in \partial_{p} Q_{\eta}$ because the process $\widehat{S}$ is almost surely continuous. Therefore,

$$
\left(v^{M_{2}}\right)^{*}(\theta, \widehat{S}(\theta)) \leqslant \varphi^{k}(\theta, \widehat{S}(\theta))-4 \eta .
$$

4. Set

$$
\begin{aligned}
& \left(\hat{\tau}_{0}, \hat{\tau}_{1}, \hat{\tau}_{2}\right)=(\hat{t}, \theta, T), \quad \hat{y}^{0}:=D \varphi(\hat{t}, \hat{s}), \quad \hat{y}^{1}:=0, \\
& \hat{\alpha}(r):=\mathbf{1}_{\{\hat{t} \leqslant r<\theta\}} \mathcal{L} D \varphi^{k}(r, \widehat{S}(r)) \quad \text { and } \quad \hat{\gamma}(r):=\mathbf{1}_{\{\hat{t} \leqslant r<\theta\}}\left(D^{2} \varphi^{k}(r, \widehat{S}(r))+B\right) .
\end{aligned}
$$

Then, the corresponding control $\hat{v}$ is in $\mathcal{A}_{\hat{t}, \hat{s}}^{M_{1}}$.

5. Consider the initial capital

$$
\hat{x}:=v^{M_{3}}(\hat{t}, \hat{s})-\eta \text {. }
$$

Proceeding as in Step 4 of the proof of Theorem 4.2, we obtain that

$$
X_{\hat{t}, \hat{s}, \hat{x}}^{\hat{v}}(\theta) \geqslant v^{M_{2}}(\theta, \widehat{S}(\theta))+\eta,
$$

which is in contradiction to the partial dynamic programming result of Lemma 4.1.

Hence, there exists for all $k \geqslant 1 \mathrm{a}\left(t_{k}, s_{k}\right) \in Q_{1 / k}$ such that

$$
F\left(s_{k}, \varphi_{t}^{k}\left(t_{k}, s_{k}\right), D^{2} \varphi^{k}\left(t_{k}, s_{k}\right)+B\right) \leqslant 0,
$$

and therefore, 


$$
\begin{aligned}
& \min \left\{L\left(s_{k}, \varphi_{t}^{k}\left(t_{k}, s_{k}\right), D^{2} \varphi^{k}\left(t_{k}, s_{k}\right)+B\right) ; H\left(\operatorname{diag}\left[s_{k}\right]\left(D^{2} \varphi^{k}\left(t_{k}, s_{k}\right)+B\right) \operatorname{diag}\left[s_{k}\right]\right)\right\} \\
& \quad=\min \left\{k-\frac{1}{2} \operatorname{Tr}\left[\sigma\left(s_{k}\right)^{T} \operatorname{diag}\left[s_{k}\right]\left(D^{2}\left\{\psi\left(s_{k}\right)+\left|s_{k}-s_{0}\right|^{4}\right\}+B\right) \operatorname{diag}\left[s_{k}\right] \sigma\left(s_{k}\right)\right] ;\right. \\
& \left.H\left(\operatorname{diag}\left[s_{k}\right]\left(D^{2}\left\{\psi\left(s_{k}\right)+\left|s_{k}-s_{0}\right|^{4}\right\}+B\right) \operatorname{diag}\left[s_{k}\right]\right)\right\} \leqslant 0 .
\end{aligned}
$$

This shows that for large enough $k$,

$$
H\left(\operatorname{diag}\left[s_{k}\right]\left(D^{2}\left\{\psi\left(s_{k}\right)+\left|s_{k}-s_{0}\right|^{4}\right\}+B\right) \operatorname{diag}\left[s_{k}\right]\right) \leqslant 0 .
$$

It follows that

$$
H\left(\operatorname{diag}\left[s_{0}\right]\left(D^{2} \psi\left(s_{0}\right)+B\right) \operatorname{diag}\left[s_{0}\right]\right) \leqslant 0,
$$

and since $B$ was arbitrary,

$\widehat{H}\left(\operatorname{diag}\left[s_{0}\right] D^{2} \psi\left(s_{0}\right) \operatorname{diag}\left[s_{0}\right]\right) \leqslant 0$.

\subsection{Upper bound under (3.9)}

Proposition 6.4. Assume (3.9). Then there exists a constant $C$ such that

$$
\bar{v}(t, s) \leqslant \hat{g}(s)+C(T-t), \quad \text { for all }(t, s) \in[0, T] \times[0, \infty)^{d} .
$$

Proof. Fix a $\left(t_{0}, s_{0}\right) \in[0, T) \times(0, \infty)^{d}$. By Lemma 3.5.b, the function $\hat{g}-U$ is concave, where $U(s)=$ $-\sum_{j=1}^{d} \gamma_{j}^{*} \log s_{j}$. Therefore, there exists a vector $z \in \mathbb{R}^{d}$ such that

$$
\hat{g}(s)-U(s) \leqslant \hat{g}\left(s_{0}\right)-U\left(s_{0}\right)+z^{T}\left(s-s_{0}\right) \text { for all } s \in(0, \infty)^{d} .
$$

Hence, the function

$$
f(s):=\hat{g}\left(s_{0}\right)-U\left(s_{0}\right)+U(s)+z^{T}\left(s-s_{0}\right)
$$

dominates $\hat{g}$. Since $0 \leqslant \hat{g} \leqslant G$, there exists a $C^{\infty}$-approximation $\tilde{f}$ of $f$ that dominates $\hat{g}$ such that $\tilde{f}\left(s_{0}\right)=\hat{g}\left(s_{0}\right)$, all derivatives of $\tilde{f}$ are bounded and

$$
\operatorname{diag}[s] D^{2} \tilde{f}(s) \operatorname{diag}[s] \in K
$$

for all $s \in(0, \infty)^{d}$. Now, let the control $v$ be given by

$$
\begin{aligned}
& \left(\tau_{0}, \tau_{1}\right):=\left(t_{0}, T\right), \quad y^{0}:=D \tilde{f}\left(s_{0}\right), \\
& \alpha(r):=\mathcal{L} D \tilde{f}\left(S_{t_{0}, s_{0}}(r)\right), \quad \gamma(r):=D^{2} \tilde{f}\left(S_{t_{0}, s_{0}}(r)\right) \quad \text { for } r \in\left[t_{0}, T\right] .
\end{aligned}
$$

Then, by twice applying Itô's lemma, we obtain for all $x_{0} \geqslant 0$,

$$
\begin{aligned}
& X_{t_{0}, s_{0}, x_{0}}^{v}(T)-\tilde{f}\left(S_{t_{0}, s_{0}}(T)\right) \\
& =x_{0}-\tilde{f}\left(s_{0}\right)-\int_{t_{0}}^{T} \frac{1}{2} \operatorname{Tr}\left[\sigma\left(S_{t_{0}, s_{0}}(r)\right)^{T} \operatorname{diag}\left[S_{t_{0}, s_{0}}(r)\right] D^{2} \tilde{f}\left(S_{t_{0}, s_{0}}(r)\right) \operatorname{diag}\left[S_{t_{0}, s_{0}}(r)\right] \sigma\left(S_{t_{0}, s_{0}}(r)\right)\right] \mathrm{d} r \\
& \geqslant x_{0}-\tilde{f}\left(s_{0}\right)-\int_{t_{0}}^{T} \frac{1}{2} \operatorname{Tr}\left[\sigma^{T}\left(S_{t_{0}, s_{0}}(r)\right) \operatorname{diag}\left[\gamma^{*}\right] \sigma\left(S_{t_{0}, s_{0}}(r)\right)\right] \mathrm{d} r \\
& \geqslant x_{0}-\tilde{f}\left(s_{0}\right)-C\left(T-t_{0}\right)
\end{aligned}
$$

for some constant $C$ that does not depend on $\left(t_{0}, s_{0}\right)$. For $x_{0}=\tilde{f}\left(s_{0}\right)+C\left(T-t_{0}\right)$, we get

$$
X_{t_{0}, s_{0}, x_{0}}^{\nu}(T) \geqslant \tilde{f}\left(S_{t_{0}, s_{0}}(T)\right) \geqslant \hat{g}\left(S_{t_{0}, s_{0}}(T)\right) \geqslant g\left(S_{t_{0}, s_{0}}(T)\right) .
$$


Since all the derivatives of $\tilde{f}$ are bounded, there exists a constant $M \geqslant 1$ such that $v \in \mathcal{A}_{t_{0}, s_{0}}^{M}$ and

$$
v^{M}\left(t_{0}, s_{0}, y_{0}\right) \leqslant x_{0}=\tilde{f}\left(s_{0}\right)+C\left(T-t_{0}\right)=\hat{g}\left(s_{0}\right)+C\left(T-t_{0}\right) .
$$

An inspection of the above argument shows that there exists a neighborhood $\mathcal{N}$ of $\left(t_{0}, s_{0}\right)$ and a constant $\hat{M} \geqslant M$ such that

$$
v^{\hat{M}}(t, s) \leqslant \hat{g}(s)+C(T-t),
$$

for all $(t, s) \in \mathcal{N}$. This implies

$$
\bar{v}(t, s) \leqslant \hat{g}(s)+C(T-t)
$$

for all $(t, s) \in[0, T) \times(0, \infty)^{d}$, and the proposition is proved.

\section{Comparison result}

In this section, we prove the comparison result, Proposition 3.9. Although this comparison is valid for more general nonlinear equations, we work here with the specific equation under consideration to simplify the presentation.

Our proof uses standard techniques from the theory of viscosity solutions. We start by recalling the notion of a strict viscosity supersolution. We will then establish the comparison result when the viscosity supersolution is strict, and deduce Proposition 3.9 from there.

\subsection{Strict viscosity supersolutions}

Definition 7.1. For a non-negative constant $\eta$, we say that a function $w$ is an $\eta$-strict viscosity supersolution of Eq. (3.4) if

$$
\widehat{F}\left(s_{0}, \varphi_{t}\left(t_{0}, s_{0}\right), D^{2} \varphi\left(t_{0}, s_{0}\right)\right)>\eta,
$$

for all $\left(t_{0}, s_{0}\right) \in[0, T) \times(0, \infty)^{d}$ and $\varphi \in C^{\infty}\left([0, T] \times[0, \infty)^{d}\right)$ such that

$$
0=\left(w_{*}-\varphi\right)\left(t_{0}, s_{0}\right)=\min _{(t, s) \in[0, T] \times[0, \infty)^{d}}\left(w_{*}-\varphi\right)(t, s) .
$$

In Section II.4 in [8], it is shown that an equivalent definition is obtained by allowing $\left(t_{0}, s_{0}\right)$ to be a local instead of a global minimum of $w_{*}-\varphi$.

The first step in the proof of Proposition 3.9 is to find a strict viscosity supersolution of Eq. (3.4).

Lemma 7.2. Assume (3.6). Then, the function

$$
w^{1}(t, s):=(T-t)+c^{*}\left[1+s_{1}+\cdots+s_{d}\right]
$$

is an $\eta^{1}$-strict viscosity supersolution of Eq. (3.4) for some $\eta^{1}>0$, and $w^{1} \geqslant \hat{g}$.

Proof. The inequality $w^{1} \geqslant \hat{g}$ follows from (3.7). It follows from (2.7) that $H(0)>0$, and therefore,

$$
\widehat{F}\left(s, w_{t}^{1}(t, s), D^{2} w^{1}(t, s)\right) \geqslant F\left(s, w_{t}^{1}(t, s), D^{2} w^{1}(t, s)\right)=\min \{1, H(0)\}=: 2 \eta^{1}>0 .
$$

Lemma 7.3. Let $w^{0}$ be a lower semicontinuous viscosity supersolution of the equation

$$
\widehat{F}\left(s, w_{t}^{0}(t, s), D^{2} w^{0}(t, s)\right)=0 .
$$

Furthermore, let $w^{1}$ be a lower semicontinuous $\eta$-strict viscosity supersolution of Eq. (7.1) for some $\eta \geqslant 0$.

Then, for all $\mu \in(0,1)$, the function $w^{\mu}:=(1-\mu) w^{0}+\mu w^{1}$ is a $\mu \eta$-strict viscosity supersolution of Eq. (7.1). 
Proof. We only need and prove this result for the case where $w^{1} \in C^{2}\left([0, T) \times(0, \infty)^{d}\right)$. The general case can be treated as in [2, page 39]. For $\mu \in(0,1)$, let $\left(t_{0}, s_{0}\right) \in[0, T) \times(0, \infty)^{d}$ and $\varphi \in C^{\infty}\left([0, T] \times[0, \infty)^{d}\right)$ such that

$$
0=\left(w^{\mu}-\varphi\right)\left(t_{0}, s_{0}\right)=\min _{(t, s) \in[0, T] \times[0, \infty)^{d}}\left(w^{\mu}-\varphi\right)(t, s) .
$$

Then, $\left(t_{0}, s_{0}\right)$ is a minimizer of the difference $w^{0}-\psi$, where

$$
\psi:=(1-\mu)^{-1}\left(\varphi-\mu w^{1}\right) .
$$

Therefore,

$$
\widehat{F}\left(s_{0}, \psi_{t}\left(t_{0}, s_{0}\right), D^{2} \psi\left(t_{0}, s_{0}\right)\right) \geqslant 0
$$

because $w^{0}$ is a viscosity supersolution of (7.1). Note that $\varphi=(1-\mu) \psi+\mu w^{1}$, and by Lemma 3.3, the function $\widehat{F}\left(s_{0}, \cdot, \cdot\right)$ is concave. Therefore,

$$
\begin{aligned}
\widehat{F}\left(s_{0}, \varphi_{t}\left(t_{0}, s_{0}\right), D^{2} \varphi\left(t_{0}, s_{0}\right)\right) & \geqslant(1-\mu) \widehat{F}\left(s_{0}, \psi_{t}\left(t_{0}, s_{0}\right), D^{2} \psi\left(t_{0}, s_{0}\right)\right)+\mu \widehat{F}\left(s_{0}, w_{t}^{1}\left(t_{0}, s_{0}\right), D^{2} w^{1}\left(t_{0}, s_{0}\right)\right) \\
& >\mu \eta . \quad \square
\end{aligned}
$$

\subsection{Proof of the comparison result}

Proposition 7.4. Assume (3.6). Suppose $u$ is an upper semicontinuous viscosity subsolution of (3.4) and $w$ a lower semicontinuous $\eta$-strict viscosity supersolution of (3.4) for some $\eta>0$. Furthermore, assume that there exists a constant $C$ so that

$$
u(t, s) \leqslant \hat{g}(s)+C \quad \text { and } \quad w(t, s) \geqslant \hat{g}(s)-C \quad \text { for all }(t, s) \in[0, T) \times(0, \infty)^{d} .
$$

Then, $u(T, \cdot) \leqslant \hat{g}(\cdot) \leqslant w(T, \cdot)$ implies that $u(t, s) \leqslant w(t, s)$ on $[0, T) \times(0, \infty)^{d}$.

Before proceeding to the proof of this proposition, let us show how it allows to complete the proof of the main comparison result.

Proof of Proposition 3.9. We use the technique of the proof of Theorem 2.7 in [2, page 38]. Let $u$ be an upper semicontinuous viscosity subsolution of (3.4) such that

$$
u(T, \cdot) \leqslant \hat{g}(\cdot) \text { and } u(t, s) \leqslant \hat{g}(s)+C \quad \text { on }[0, T) \times(0, \infty)^{d},
$$

and $w$ a lower semicontinuous viscosity supersolution of (3.4) such that

$$
w(T, \cdot) \geqslant \hat{g}(\cdot) \quad \text { and } \quad w(t, s) \geqslant \hat{g}(s)-C \quad \text { on }[0, T) \times(0, \infty)^{d} .
$$

Let $w^{1}$ be the $\eta^{1}$-strict viscosity supersolution of (3.4) defined in Lemma 7.2. Then, it follows from Lemma 7.3 that

$$
w^{\mu}:=(1-\mu) w+\mu w^{1}
$$

is a $\mu \eta^{1}$-strict viscosity supersolution of (3.4) satisfying

$$
w^{\mu}(T, s) \geqslant \hat{g}(s) \quad \text { and } \quad w^{\mu}(t, s) \geqslant \hat{g}(s)-(1-\mu) C \quad \text { for all }(t, s) \in[0, T) \times(0, \infty)^{d} .
$$

We are then in the context of Proposition 7.4 and can conclude that $w^{\mu}(t, s) \geqslant u(t, s)$ on $[0, T) \times(0, \infty)^{d}$. The required result follows by sending $\mu$ to zero.

In preparation of the proof of Proposition 7.4, we provide the following technical conditions satisfied by $\widehat{F}$. 
Lemma 7.5. There exist a positive constant $C$ and a function $h:[0, \infty] \rightarrow[0, \infty]$ with $\lim _{x \rightarrow 0} h(x)=0$ such that

$$
\begin{aligned}
& \left|\widehat{F}(s, p, A)-\widehat{F}\left(s, p^{\prime}, A\right)\right| \leqslant\left|p-p^{\prime}\right|, \\
& \widehat{F}(s, p, A)-\widehat{F}(s, p, A+B) \leqslant C \operatorname{Tr}[\operatorname{diag}[s] B \operatorname{diag}[s]], \\
& \widehat{F}\left(s^{\prime}, \alpha\left(t-t^{\prime}\right), A^{\prime}\right)-\widehat{F}\left(s, \alpha\left(t-t^{\prime}\right), A\right) \leqslant h\left(\alpha\left|s-s^{\prime}\right|^{2}+\left|s-s^{\prime}\right|\right),
\end{aligned}
$$

for all $(t, s),\left(t^{\prime}, s^{\prime}\right) \in[0, T) \times(0, \infty)^{d}, \alpha>1, p \in \mathbb{R}, B \in \mathcal{S}_{+}^{d}$, and $\left(A, A^{\prime}\right) \in \mathcal{S}^{d} \times \mathcal{S}^{d}$ satisfying

$$
-3 \alpha\left(\begin{array}{cc}
I_{d} & 0 \\
0 & I_{d}
\end{array}\right) \leqslant\left(\begin{array}{cc}
A & 0 \\
0 & -A^{\prime}
\end{array}\right) \leqslant 3 \alpha\left(\begin{array}{cc}
I_{d} & -I_{d} \\
-I_{d} & I_{d}
\end{array}\right) .
$$

Proof. These conditions are classical in the theory of viscosity solutions, and are satisfied by a large class of second order nonlinear partial differential equations, see [5]. The inequalities (7.3) and (7.4) can be verified directly. Inequality (7.5) can be shown as in Example 3.6 of the User's Guide [5].

Proof of Proposition 7.4. We adapt the general procedure reported in [5].

1. For $\varepsilon, \alpha>0$, consider the upper semicontinuous function

$$
\Phi^{(\varepsilon, \alpha)}\left(t, t^{\prime}, s, s^{\prime}\right):=u(t, s)-w\left(t^{\prime}, s^{\prime}\right)-\varepsilon\left(l(s)+l\left(s^{\prime}\right)\right)-\frac{1}{2} \alpha\left(\left(t-t^{\prime}\right)^{2}+\left(s-s^{\prime}\right)^{2}\right),
$$

where

$$
l(s):=\sum_{j=1}^{d}\left[s_{j}-\log s_{j}\right],
$$

and set

$$
\phi^{\varepsilon}(t, s):=\Phi^{(\varepsilon, \alpha)}(t, t, s, s)
$$

(note that $\phi^{\varepsilon}$ is independent of $\alpha$ ). In view of (7.2),

$$
\phi^{\varepsilon}(t, s)=u(t, s)-w(t, s)-2 \varepsilon l(s) \leqslant 2 C-2 \varepsilon l(s),
$$

which shows that

$$
\max _{[0, T] \times[0, \infty)^{d}} \phi^{\varepsilon}(t, s)=\phi^{\varepsilon}\left(t_{\varepsilon}, s_{\varepsilon}\right) \quad \text { for some }\left(t_{\varepsilon}, s_{\varepsilon}\right) \in[0, T] \times(0, \infty)^{d} .
$$

In Step 2 below, we will prove that

$$
t_{\varepsilon_{k}}=T \text { for some sequence }\left(\varepsilon_{k}\right)_{k} \geqslant 1 \text { with } \varepsilon_{k}>0 \text { and } \varepsilon_{k} \rightarrow 0 .
$$

Using this, we arrive at

$$
\begin{aligned}
u(t, s)-w(t, s) & =\phi^{\varepsilon_{k}}(t, s)+2 \varepsilon_{k} l(s) \leqslant \phi^{\varepsilon_{k}}\left(T, s_{\varepsilon_{k}}\right)+2 \varepsilon_{k} l(s) \\
& =u\left(T, s_{\varepsilon_{k}}\right)-w\left(T, s_{\varepsilon_{k}}\right)-2 \varepsilon_{k} l\left(s_{\varepsilon_{k}}\right)+2 \varepsilon_{k} l(s) \\
& \leqslant u\left(T, s_{\varepsilon_{k}}\right)-w\left(T, s_{\varepsilon_{k}}\right)+2 \varepsilon_{k} l(s)
\end{aligned}
$$

by the non-negativity of $l$. Since $u(T, \cdot) \leqslant w(T, \cdot)$, this shows that

$$
u(t, s)-w(t, s) \leqslant 2 \varepsilon_{k} l(s) \quad \text { for all }(t, s) \in[0, T) \times(0, \infty)^{d},
$$

and the required result follows by sending $k$ to infinity.

2. In order to prove (7.7), we assume to the contrary that there is a constant $\bar{\varepsilon}>0$ such that

$$
t_{\varepsilon}<T \text { for all } 0<\varepsilon \leqslant \bar{\varepsilon},
$$


and we work towards a contradiction.

Set

$$
\widehat{\Phi}^{(\varepsilon, \alpha)}\left(t, t^{\prime}, s, s^{\prime}\right):=\Phi^{\varepsilon, \alpha}\left(t, t^{\prime}, s, s^{\prime}\right)-\frac{1}{2}\left[\left(t-t_{\varepsilon}\right)^{2}+\left(t^{\prime}-t_{\varepsilon}\right)^{2}\right]-\frac{1}{4}\left[\left|s-s_{\varepsilon}\right|^{4}+\left|s^{\prime}-s_{\varepsilon}\right|^{4}\right],
$$

and $\hat{\phi}^{\varepsilon}(t, s):=\widehat{\Phi}^{(\varepsilon, \alpha)}(t, t, s, s)$. Then, $\left(t_{\varepsilon}, s_{\varepsilon}\right)$ is a strict maximizer of $\hat{\phi}^{\varepsilon}$. By Proposition 3.7 in [5], for every $\varepsilon \leqslant \bar{\varepsilon}$, there exists a sequence $\alpha_{k} \rightarrow \infty$ and local maximizers $\left(t_{k}, t_{k}^{\prime}, s_{k}, s_{k}^{\prime}\right)$ of $\widehat{\Phi}^{\left(\varepsilon, \alpha_{k}\right)}$ satisfying

$$
\left(t_{k}, t_{k}^{\prime}, s_{k}, s_{k}^{\prime}\right) \longrightarrow\left(t_{\varepsilon}, t_{\varepsilon}, s_{\varepsilon}, s_{\varepsilon}\right) \quad \text { as } k \rightarrow \infty
$$

and

$$
\alpha_{k}\left(\left(t_{k}-t_{k}^{\prime}\right)^{2}+\left(s_{k}-s_{k}^{\prime}\right)^{2}\right) \rightarrow 0 \quad \text { as } k \rightarrow \infty
$$

In particular, for $\varepsilon$ sufficiently small and $\alpha_{k}$ sufficiently large, $t_{k}<T$ and $t_{k}^{\prime}<T$. We now apply Theorem 3.2 in [5] to the sequence of local maxima $\left(t_{k}, t_{k}^{\prime}, s_{k}, s_{k}^{\prime}\right)$ of $\widehat{\Phi}^{\left(\varepsilon, \alpha_{k}\right)}$. Then, for sufficiently large $\alpha_{k}$, there exist two symmetric matrices $A_{k}, A_{k}^{\prime} \in \mathcal{S}^{d}$ such that,

$$
\begin{aligned}
& \left(A_{k}, A_{k}^{\prime}\right) \text { satisfies }(7.6), \\
& \left(p_{k}+\left(t_{k}-t_{\varepsilon}\right), q_{k}, A_{k}+\varepsilon D^{2} l\left(s_{k}\right)+Q\left(s_{k}-s_{\varepsilon}\right)\right) \in \bar{J}^{2,-} u\left(t_{k}, s_{k}\right), \\
& \left(p_{k}-\left(t_{k}^{\prime}-t_{\varepsilon}\right), q_{k}^{\prime}, A_{k}^{\prime}-\varepsilon D^{2} l\left(s_{k}^{\prime}\right)-Q\left(s_{k}^{\prime}-s_{\varepsilon}\right)\right) \in \bar{J}^{2,+} w\left(t_{k}^{\prime}, s_{k}^{\prime}\right),
\end{aligned}
$$

where

$$
\begin{aligned}
& p_{k}:=\alpha_{k}\left(t_{k}-t_{k}^{\prime}\right), \quad Q(z)=2 z \otimes z+|z|^{2} I_{d}, \\
& q_{k}:=\alpha_{k}\left(s_{k}-s_{k}^{\prime}\right)+\left(s_{k}-s_{\varepsilon}\right)\left|s_{k}-s_{\varepsilon}\right|^{2}+\varepsilon \operatorname{Dl}\left(s_{k}\right), \\
& q_{k}^{\prime}:=\alpha_{k}\left(s_{k}-s_{k}^{\prime}\right)-\left(s_{k}^{\prime}-s_{\varepsilon}\right)\left|s_{k}^{\prime}-s_{\varepsilon}\right|^{2}-\varepsilon \operatorname{Dl}\left(s_{k}^{\prime}\right) .
\end{aligned}
$$

Here, $\bar{J}^{2,+} v(t, s)$ and $\bar{J}^{2,-} v(t, s)$ denote the closed second order superjet and subjet of the function $v$ at the point $(t, s)$, see [5] for the definition.

Since $u$ is a viscosity subsolution of (3.4),

$$
\widehat{F}\left(s_{k}, p_{k}+\left(t_{k}-t_{\varepsilon}\right), A_{k}+\varepsilon D^{2} l\left(s_{k}\right)+Q\left(s_{k}-s_{\varepsilon}\right)\right) \leqslant 0 .
$$

Also, the positivity of $D^{2} l$ and $Q$, together with (7.3) and (7.4), imply

$$
\begin{aligned}
\widehat{F}\left(s_{k}, p_{k}, A_{k}\right) \leqslant & \widehat{F}\left(s_{k}, p_{k}+\left(t_{k}-t_{\varepsilon}\right), A_{k}+\varepsilon D^{2} l\left(s_{k}\right)+Q\left(s_{k}-s_{\varepsilon}\right)\right) \\
& +\left|t_{k}-t_{\varepsilon}\right|+C \operatorname{Tr}\left(\operatorname{diag}\left[s_{k}\right]\left[\varepsilon D^{2} l\left(s_{k}\right)+Q\left(s_{k}-s_{\varepsilon}\right)\right] \operatorname{diag}\left[s_{k}\right]\right), \\
\leqslant & \left|t_{k}-t_{\varepsilon}\right|+\widehat{C}\left[\varepsilon+\left|s_{k}-s_{\varepsilon}\right|^{2}\left|s_{k}\right|^{2}\right],
\end{aligned}
$$

for some constant $\widehat{C}$. In the last step we used the fact that $\operatorname{diag}[s] D^{2} l(s) \operatorname{diag}[s]=I_{d}$, the identity matrix.

We proceed as above and use the fact that $w$ is an $\eta$-strict viscosity supersolution of (3.4). The result is,

$$
\widehat{F}\left(s_{k}^{\prime}, p_{k}, A_{k}^{\prime}\right) \geqslant \eta-\left|t_{k}^{\prime}-t_{\varepsilon}\right|-\widehat{C}\left[\varepsilon+\left|s_{k}^{\prime}-s_{\varepsilon}\right|^{2}\left|s_{k}^{\prime}\right|^{2}\right] .
$$

Combining the last two inequalities, we get

$$
\widehat{F}\left(s_{k}^{\prime}, p_{k}, A_{k}^{\prime}\right)-\widehat{F}\left(s_{k}, p_{k}, A_{k}\right) \geqslant \eta-\left|t_{k}-t_{\varepsilon}\right|-\left|t_{k}^{\prime}-t_{\varepsilon}\right|-\widehat{C}\left[2 \varepsilon+\left|s_{k}-s_{\varepsilon}\right|^{2}\left|s_{k}\right|^{2}+\left|s_{k}^{\prime}-s_{\varepsilon}\right|^{2}\left|s_{k}^{\prime}\right|^{2}\right] .
$$

Therefore,

$$
\liminf _{k \rightarrow \infty}\left[\widehat{F}\left(s_{k}^{\prime}, p_{k}, A_{k}^{\prime}\right)-\widehat{F}\left(s_{k}, p_{k}, A_{k}\right)\right] \geqslant \eta-2 \widehat{C} \varepsilon
$$


On the other hand, since $A_{k}, A_{k}^{\prime}$ satisfy (7.6), it follows from (7.5) that

$$
\widehat{F}\left(s_{k}^{\prime}, p_{k}, A_{k}^{\prime}\right)-\widehat{F}\left(s_{k}, p_{k}, A_{k}\right) \leqslant h\left(\alpha_{k}\left[\left|s_{k}-s_{k}^{\prime}\right|^{2}\right]+\left|s_{k}-s_{k}^{\prime}\right|\right) .
$$

Since $\alpha_{k}\left[\left|s_{k}-s_{k}^{\prime}\right|^{2}\right]+\left|s_{k}-s_{k}^{\prime}\right|$ tends to zero as $k$ approaches infinity, (7.9) is in contradiction to (7.8) for $\varepsilon<$ $\eta /(2 \widehat{C})$. Hence, (7.7) has to hold.

Remark 7.6. Note that, when $g$ is continuous, the above comparison proof also applies to Eq. (3.5):

$$
\min \left\{f(s)-g(s), \widehat{H}\left(\operatorname{diag}[s] D^{2} f(s) \operatorname{diag}[s]\right)\right\}=0,
$$

and then yields that if $u$ is a viscosity subsolution and $w$ is a viscosity supersolution such that $u-w$ is bounded, then $u \leqslant w$.

\section{Acknowledgements}

We are grateful to Peter Bank for pointing out the approximation results of [1] and [13]. The comparison of our results with these references led to the discussion in Remark 3.11.

\section{Appendix. Small time behavior of double stochastic integrals}

We here report results from [4] on the small time path behavior of double stochastic integrals that are needed in Section 5. In this appendix, $\{W(t), t \geqslant 0\}$ is a $d$-dimensional Brownian motion on a filtered probability space $(\Omega, \mathcal{F}, \mathbb{F}=\{\mathcal{F}(t), t \geqslant 0\}, P)$ satisfying the usual conditions. The filtration $\mathbb{F}$ can be bigger than the smallest filtration that satisfies the usual conditions and contains the filtration generated by $W$.

For $B \in \mathcal{S}^{d}$, we denote by $\lambda^{*}(B)$ the largest eigenvalue of $B$. Note that $\lambda^{*}$ is a continuous, and therefore, measurable function from $\mathcal{S}^{d}$ to $\mathbb{R}$.

Theorem A.1. Let $\{M(t), t \geqslant 0\}$ be an $\mathbb{R}^{d}$-valued martingale defined by

$$
M(t):=\int_{0}^{t} m(r) \mathrm{d} W(r), \quad t \geqslant 0,
$$

where $\{m(t), t \geqslant 0\}$ is a bounded, $\mathcal{M}^{d}$-valued, $\mathbb{F}$-progressively measurable process such that there exists a random variable $\varepsilon>0$ so that almost surely,

$$
\int_{0}^{t}|m(r)-m(0)|^{2} \mathrm{~d} r=\mathrm{O}\left(t^{1+\varepsilon}\right) \text { for } t \searrow 0 .
$$

(a) Let $\{b(t), t \geqslant 0\}$ be a bounded, $\mathcal{M}^{d}$-valued, $\mathbb{F}$-progressively measurable process such that for all $t \geqslant 0$, $\left|m(0)^{T} b(t) m(0)\right| \leqslant 1$. Then

$$
\underset{t \searrow 0}{\limsup } \frac{1}{t \log \log (1 / t)}\left|\int_{0}^{t}\left(\int_{0}^{u} b(u) \mathrm{d} M(u)\right)^{T} \mathrm{~d} M(r)\right| \leqslant 1 .
$$


(b) Let $B$ be a bounded, $\mathcal{F}(0)$-measurable, $\mathcal{S}^{d}$-valued random variable with $\lambda^{*}(B) \geqslant 0$. If $\{b(t), t \geqslant 0\}$ is a bounded, $\mathcal{S}^{d}$-valued, $\mathbb{F}$-progressively measurable process such that for all $t \geqslant 0$,

$$
m(0)^{T} b(t) m(0) \geqslant B,
$$

then

$$
\limsup _{t \searrow 0} \frac{1}{t \log \log (1 / t)} \int_{0}^{t}\left(\int_{0}^{r} b(u) \mathrm{d} M(u)\right)^{T} \mathrm{~d} M(r) \geqslant \lambda^{*}(B) .
$$

Theorem A.2. Let $\{M(t), t \geqslant 0\}$ be an $\mathbb{R}^{d}$-valued martingale defined by

$$
M(t)=\int_{0}^{t} m(r) \mathrm{d} W(r), \quad t \geqslant 0,
$$

where $\{m(t), t \geqslant 0\}$ is a bounded, $\mathcal{M}^{d}$-valued, $\mathbb{F}$-progressively measurable process. Let $\{b(t), t \geqslant 0\}$ be a bounded, $\mathcal{M}^{d}$-valued, $\mathbb{F}$-progressively measurable process with $b(0) \in \mathcal{S}^{d}$, and assume that there exists a random variable $\varepsilon>0$ such that almost surely,

$$
\int_{0}^{t}|m(r)-m(0)|^{2} \mathrm{~d} r=\mathrm{O}\left(t^{1+\varepsilon}\right) \quad \text { and } \int_{0}^{t}|b(r)-b(0)|^{2} \mathrm{~d} r=\mathrm{O}\left(t^{1+\varepsilon}\right) \quad \text { for } t \searrow 0 .
$$

If $m(0)^{T} b(0) m(0) \leqslant 0$, then

$$
\underset{t \searrow 0}{\limsup } \frac{2}{t} \int_{0}^{t}\left(\int_{0}^{r} b(u) \mathrm{d} M(u)\right)^{T} \mathrm{~d} M(r)=-\operatorname{Tr}\left[m(0)^{T} b(0) m(0)\right] .
$$

Proposition A.3. Let $\{a(t), t \geqslant 0\}$ be a bounded, $\mathbb{R}^{d}$-valued, $\mathbb{F}$-progressively measurable process and $\{m(t)$, $t \geqslant 0$ an $\mathcal{M}^{d}$-valued, $\mathbb{F}$-progressively measurable process such that

$$
\int_{0}^{t}|m(r)|^{2} \mathrm{~d} r<\infty \quad \text { for all } t \geqslant 0,
$$

and there exists a $(0,1]$-valued random variable $\varepsilon$ such that almost surely,

$$
\int_{0}^{t} r^{2}|m(r)|^{2} \mathrm{~d} r=\mathrm{O}\left(t^{3-\varepsilon}\right) \quad \text { for } t \searrow 0 .
$$

Then,

$$
\lim _{t \searrow 0} t^{-3 / 2+\varepsilon} \int_{0}^{t}\left(\int_{0}^{r} a(u) \mathrm{d} u\right)^{T} m(r) \mathrm{d} W(r)=0 .
$$

\section{References}

[1] P. Bank, D. Baum, Hedging and portfolio optimization in financial markets with a large trader, Math. Finance 14 (1) (2004) 1-18.

[2] G. Barles, Solutions de Viscosité des Equations de Hamilton-Jacobi, Math. Appl., vol. 17, Springer-Verlag, Paris, 1994. 
[3] M. Broadie, J. Cvitanić, H.M. Soner, Optimal replication of contingent claims under portfolio constraints, Rev. Financial Stud. 11 (1998) 59-79.

[4] P. Cheridito, H.M. Soner, N. Touzi, Small time path behavior of double stochastic integrals and applications to stochastic control, Preprint, 2003.

[5] M.G. Crandall, H. Ishii, P.L. Lions, User's guide to viscosity solutions of second order partial differential equations, Bull. Amer. Math. Soc. 27 (1) (1992) 1-67.

[6] J. Cvitanić, I. Karatzas, Hedging contingent claims with constrained portfolios, Ann. Appl. Probab. 3 (3) (1993) 652-681.

[7] J. Cvitanić, H. Pham, N. Touzi, Super-replication in stochastic volatility models under portfolio constraints, J. Appl. Probab. 36 (2) (1999) 523-545.

[8] W.H. Fleming, H.M. Soner, Controlled Markov Processes and Viscosity Solutions, Math. Appl., vol. 25, Springer-Verlag, New York, 1993.

[9] H. Föllmer, D. Kramkov, Optional decomposition under constraints, Probab. Theory Related Fields 109 (1) (1997) 1-25.

[10] E. Jouini, H. Kallal, Arbitrage in securities markets with short-sales constraints, Math. Finance 5 (3) (1995) 197-232.

[11] I. Karatzas, S. Shreve, Methods of Mathematical Finance, Math. Appl., vol. 39, Springer-Verlag, New York, 1998.

[12] T.G. Kurtz, P. Protter, Weak convergence of stochastic integrals and differential equations, in: D. Talay, L. Tubaro (Eds.), Probabilistic Models for Nonlinear Partial Differential Equations, Montecatini Terme, 1995, in: Lecture Notes in Math., vol. 1629, 1996, pp. 1-41.

[13] S. Levental, A.V. Skorohod, On the possibility of hedging options in the presence of transaction costs, Ann. Appl. Probab. 7 (2) (1997) 410-443.

[14] D. Revuz, M. Yor, Continuous Martingales and Brownian Motion, third ed., Springer-Verlag, Berlin, 1999.

[15] H.M. Soner, N. Touzi, Super-replication under gamma constraints, SIAM J. Control Optim. 39 (1) (2000) 73-96.

[16] H.M. Soner, N. Touzi, Stochastis target problem, dynamic programming, and viscosity solutions, SIAM J. Control Optim. 41 (2) (2002) 404-424.

[17] H.M. Soner, N. Touzi, Dynamic programming for stochastic target problems and geometric flows, J. Eur. Math. Soc. 4 (3) (2002) $201-236$.

[18] N. Touzi, Direct characterization of the value of super-replication under stochastic volatility and portfolio constraint, Stochastic Process. Appl. 88 (2) (2000) 305-328.

[19] N. Touzi, Stochastic Control and Application to Finance, Lecture Notes, Scuola Normale Superiore Pisa, 2002. 